\title{
Research Article \\ Classification of Single and Multiple Disturbances in Electric Signals
}

\author{
Moisés Vidal Ribeiro and José Luiz Rezende Pereira \\ Department of Electrical Energy, Federal University of Juiz de Fora, 36036330 Juiz de fora, MG, Brazil
}

Received 19 April 2006; Revised 28 January 2007; Accepted 16 May 2007

Recommended by Pradipta Kishore Dash

This paper discusses and presents a different perspective for classifying single and multiple disturbances in electric signals, such as voltage and current ones. Basically, the principle of divide to conquer is applied to decompose the electric signals into what we call primitive signals or components from which primitive patterns can be independently recognized. A technique based on such concept is introduced to demonstrate the effectiveness of such idea. This technique decomposes the electric signals into three main primitive components. In each primitive component, few high-order-statistics- (HOS-) based features are extracted. Then, Bayes' theory-based techniques are applied to verify the ocurrence or not of single or multiple disturbances in the electric signals. The performance analysis carried out on a large number of data indicates that the proposed technique outperforms the performance attained by the technique introduced by He and Starzyk. Additionally, the numerical results verify that the proposed technique is capable of offering interesting results when it is applied to classify several sets of disturbances if one cycle of the main frequency is considered, at least.

Copyright ( 92007 M. V. Ribeiro and J. L. R. Pereira. This is an open access article distributed under the Creative Commons Attribution License, which permits unrestricted use, distribution, and reproduction in any medium, provided the original work is properly cited.

\section{INTRODUCTION}

Recently, a great deal of attention has been drawn to the efficient and appropriate use of signal processing and computational intelligence techniques for the development of powerful tools to characterize, analyze, and evaluate the quality of power systems as well as the behavior of their loads. From a signal processing standpoint, the power quality (PQ) analysis could be listed in the following foremost topics: (i) disturbance detection, (ii) disturbance classification, (iii) source of disturbance identification, (iv) source of disturbance localization, (v) signal compression, (vi) parameters estimation, (vii) signal representation or decomposition, and (viii) signal and system behavior predictions.

The classification or recognition topic is an important issue for the development of the next generation of PQ monitoring equipment. Basically, it refers to the use of signal processing-based technique to extract as few as possible and, at the same time, representative features from the powerline signals, which are supposed to be voltage and current ones, followed by the use of a powerful and a simple technique to classify the detected disturbances.
As far as the use of pattern recognition technique for PQ applications has been concerned, the main reasons for developing techniques to classify disturbances are [1] (i) improvements in the tracking performance of abnormal behaviors of the monitored powerlines and electrical machines and (ii) the feasible detection of disturbance sources responsible for causing the disturbances in the monitored powerlines or electrical machines. To succeed in this aim, several techniques have been widely applied to analyze single disturbances in electric signals [2-28] in the past two decades. However, it is well recognized that during an abnormal behavior of a power system, the powerline signals are corrupted not only by single disturbance, but also by multiple ones. As a result, the majority of techniques developed so far to classify single disturbances have limited applicability in monitoring equipment since they will have to deal with multiple disturbances, even though they have not been designed to do so. Recently, in [2,3] wavelet-based classification techniques capable of classifying single and two kinds of multiple disturbances have been proposed. The results reported in [2] surpass those presented in [3] and reveal that there is a room for the development of powerful, simple, and efficient techniques to classify other sets of multiple disturbances. 
The purposes of this contribution are (i) the discussion of a formulation that facilitates the classification of single and multiple disturbances in voltage and current signals; we argue that this formulation allows the development of powerful and efficient pattern recognition techniques to classify a large number of sets of disturbances; basically, the principle of divide to conquer, which inspired the detection technique introduced in [29], is applied to decompose the electric signals into what we call primitive signals or primitive components from which primitive patterns can be recognized easily; and (ii) the discussion of a new disturbance classification technique that makes use of the proposed formulation to classify single and multiple disturbances in electric signals. This technique decomposes the electric signals into three main primitive components. In each primitive component, few high-order-statistics- (HOS-)based features are extracted. Then, effortless Bayesian classifier, which makes use of normal density function and draws on the HOS-based features, can be designed to come to light single as well as multiple disturbances. The rationale behind is that each primitive component is associated to a reduced and disjoint set of disturbances. Numerical results indicate that the proposed technique not only outperforms previous techniques, such as $[2,3]$, but also provides very interesting results in case of the frame length corresponds to at least one-cycle of the main frequency. This contribution was initially reported in [1] and partially presented in $[30,31]$.

The paper is organized as follows. Section 2 formulates the problem of single and multiple disturbances classification. Section 3 discusses the proposed technique, derived from the formulation presented in Section 2. Section 4 presents computational results indicating the improved classification performance offered by the proposed technique. Finally, concluding remarks are stated in Section 5.

\section{PROBLEM FORMULATION: SINGLE AND MULTIPLE DISTURBANCES}

The discrete version of monitored powerline signals can be divided into nonoverlapped frames of $N$ samples. The discrete sequence in a frame can be expressed as an additive contribution of several types of phenomena:

$$
x(n)=\left.x(t)\right|_{t=n T_{s}}:=f(n)+h(n)+i(n)+t(n)+v(n),
$$

where $n=0, \ldots, N-1, T_{s}=1 / f_{s}$ is the sampling period, the sequences $\{f(n)\},\{h(n)\},\{i(n)\},\{t(n)\}$, and $\{v(n)\}$ denote the power supply signal (or fundamental component), harmonics, interharmonics, transient, and background noise, respectively. Each of these signals is defined as follows:

$$
\begin{gathered}
f(n):=A_{0}(n) \cos \left[2 \pi \frac{f_{0}(n)}{f_{s}} n+\theta_{0}(n)\right], \\
h(n):=\sum_{m=1}^{M} h_{m}(n), \\
i(n):=\sum_{j=1}^{J} i_{j}(n), \\
t(n):=t_{\text {imp }}(n)+t_{\text {not }}(n)+t_{\text {cas }}(n)+t_{\text {dae }}(n),
\end{gathered}
$$

and $v(n)$ is independently and identically distributed (i.i.d.) noise as normal $\mathcal{N}\left(0, \sigma_{v}^{2}\right)$ and independent of $\{f(n)\}$, $\{h(n)\},\{i(n)\}$, and $\{t(n)\}$.

In (2), $A_{0}(n), f_{0}(n)$, and $\theta_{0}(n)$ refer to the magnitude, fundamental frequency, and phase of the power supply signal, respectively. In (3) and (4), $h_{m}(n)$ and $i_{j}(n)$ are the $m$ th harmonic and the $j$ th inter-harmonic, respectively, which are defined as

$$
\begin{aligned}
& h_{m}(n):=A_{m}(n) \cos \left[2 \pi m \frac{f_{0}(n)}{f_{s}} n+\theta_{m}(n)\right], \\
& i_{j}(n):=A_{I, j}(n) \cos \left[2 \pi \frac{f_{I, j}(n)}{f_{s}} n+\theta_{I, j}(n)\right] .
\end{aligned}
$$

In (6), $A_{m}(n)$ is the magnitude and $\theta_{m}(n)$ is the phase of the $m$ th harmonic. In (7), $A_{I, j}(n), f_{I, j}(n)$, and $\theta_{I, j}(n)$ are the magnitude, frequency, and phase of the $j$ th interharmonic, respectively. In (5), $t_{\text {imp }}(n), t_{\text {not }}(n)$, and $t_{\text {cas }}(n)$ represent impulsive transients named spikes, notches, decaying oscillations. $t_{\mathrm{dae}}(n)$ refers to oscillatory transient named damped exponentials. These transients are expressed by

$$
\begin{gathered}
t_{\mathrm{imp}}(n):=\sum_{i=1}^{N_{\mathrm{imp}}} t_{\mathrm{imp}, i}(n), \\
t_{\mathrm{not}}(n):=\sum_{i=1}^{N_{\mathrm{not}}} t_{\mathrm{not}, i}(n), \\
t_{\mathrm{dec}}(n):=\sum_{i=1}^{N_{\mathrm{dec}}} A_{\mathrm{dec}, i}(n) \cos \left[\omega_{\mathrm{dec}, i}(n) n+\theta_{\mathrm{dec}, i}(n)\right] \\
\times \exp \left[-\alpha_{\mathrm{dec}, i}\left(n-n_{\mathrm{dec}, i}\right)\right], \\
t_{\mathrm{dam}}(n):=\sum_{i=1}^{N_{\mathrm{dam}}} A_{\mathrm{dam}, i}(n) \exp \left[-\alpha_{\mathrm{dam}, i}\left(n-n_{\mathrm{dam}, i}\right)\right],
\end{gathered}
$$

respectively, where $t_{\mathrm{imp}, i}(n)$ and $t_{\mathrm{imp}, i}(n)$ are the $n$th samples of the $i$ th transient named impulsive transient or notch. Note that (10) refers to the capacitor switchings as well as signals resulted from faulted waveforms. Equation (11) defines the decaying exponential as well as direct current (DC) components $\left(\alpha_{\mathrm{dam}}=0\right)$ generated by geomagnetic disturbances, and so forth.

The following definition is used in this contribution: (i) the vector $\mathbf{x}=[x(n) \cdots x(n-N+1)]^{T}$ is composed of samples from the signal expressed by (1), the vector $\mathbf{f}=$ $[f(n) \cdots f(n-N+1)]^{T}$ constituted by estimated samples of the signal given by (2), the vector $\mathbf{h}=[h(n) \cdots h(n-$ $N+1)]^{T}$ is composed of estimated samples of the signal defined by (3), the vector $\mathbf{i}=[i(n) \cdots i(n-N+1)]^{T}$ is constituted by estimated samples of the signals defined by (4), the vector $\mathbf{t}_{\text {imp }}=\left[t_{\text {imp }}(n) \cdots t_{\text {imp }}(n-N+1)\right]^{T}$ is constituted by estimated samples of the signals defined by (8), the vector $\mathbf{t}_{\text {not }}=\left[t_{\text {not }}(n) \cdots t_{\text {not }}(n-N+1)\right]^{T}$ is constituted by estimated samples of the signals defined by (9), the vector $\mathbf{t}_{\mathrm{dec}}=\left[t_{\mathrm{dec}}(n) \cdots t_{\mathrm{dec}}(n-N+1)\right]^{T}$ is composed of estimated samples of the signals defined by (10), and the vector $\mathbf{t}_{\mathrm{dam}}=\left[t_{\mathrm{dam}}(n) \cdots t_{\mathrm{dam}}(n-N+1)\right]^{T}$ is constituted by estimated samples of the signals defined by (11). 


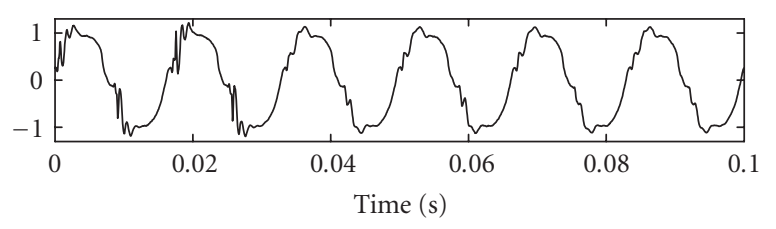

(a)

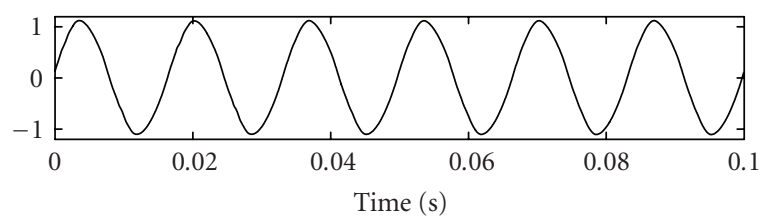

(b)

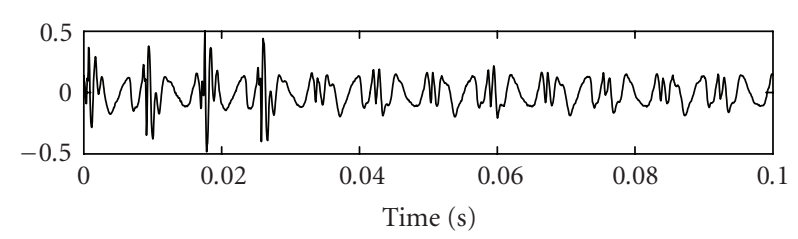

(c)

Figure 1: (a) Monitored voltage signal, $\{x(n)\}$, (b) fundamental component, $\{f(n)\},(\mathrm{c})$ harmonic and transient components, $\{h(n)\}+\{u(n)\}$.

$\mathbf{v}=[v(n) \cdots v(n-N+1)]^{T}$ is constituted by samples of the additive noise.

It is worth mentioning that low-, medium-, and highvoltage electrical networks present different sets of single and multiple disturbances. As a result, the design of classification technique for each voltage level has to take into account the information and characteristics of these networks to attain a high classification performance. For instance, the sets of disturbances in the high-voltage transmission and low-voltage distribution systems differ considerably.

The majority of classification techniques developed so far are for single disturbances. For these techniques, the feature extraction, as well as classification techniques, has been investigated and researchers in this field have achieved a great level of development [3-28]. As a result, the current classification techniques are capable of classifying single disturbances achieving classification ratio from $90 \%$ to $100 \%$. A recent technique introduced in [32] attains classification ratio very close to $100 \%$ if single disturbances are considered. The main advantage offered by this technique is the use of simple feature extraction technique along with support vector machine (SVM) technique. Nevertheless, one can note that the incidence of multiple disturbances, at the same time interval, in electric signals, is an ordinary situation owing to the presence of several sources of disturbances in the power systems. Figures 1 and 2 expose this problem very well. One can note that Figure 1(a) shows the signal $\{x(n)\}=\{f(n)\}+\{h(n)\}+\{u(n)\}+\{v(n)\}$ while Figures $1(\mathrm{~b})$ and $1(\mathrm{c})$ depict the sequences $\{f(n)\}$ and $\{x(n)\}-\{f(n)\}$, respectively. This voltage measurement was obtained from

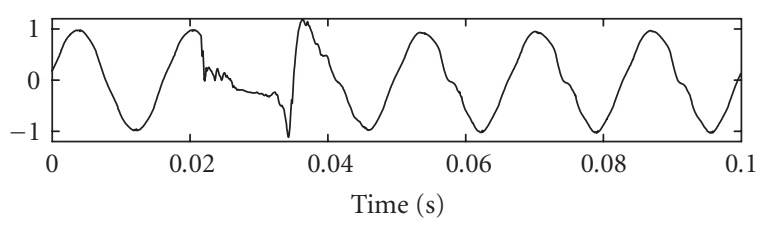

(a)

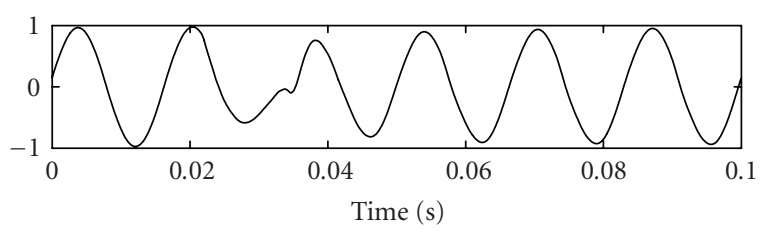

(b)

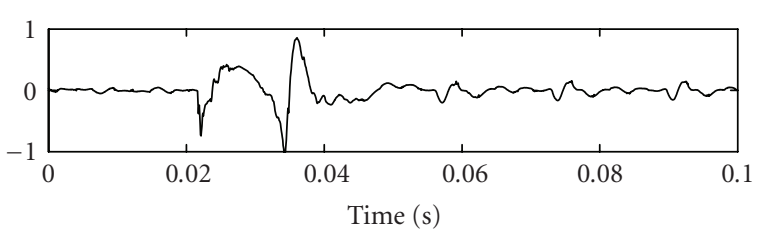

(c)

FIGURE 2: (a) Monitored voltage signal, $\{x(n)\}$, (b) fundamental component, $\{f(n)\},(c)$ harmonic and transient components, $\{h(n)\}+\{u(n)\}$.

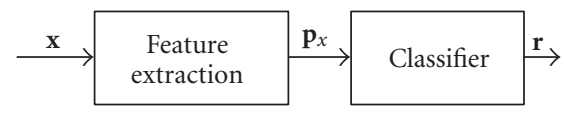

FIGURE 3: Standard paradigm for the classification of single and multiple disturbances.

IEEE working group P1159.3 website. In Figure 1(c), the signal $\{z(n)\}=\{h(n)\}+\{t(n)\}+\{v(n)\}$ is composed of 3rd harmonic, transient signal that can be a priori assumed to be a decaying oscillation, and, maybe, other disturbances very difficult to be a priori categorized. Another illustrative example of multiple disturbances in voltage signals is shown in Figure 2. One can note the incidence of short-duration voltage variation named sag, see Figure 2(b), harmonic components and, short-transient intervals associated with the voltage sag as is pictured in Figure 2(c).

Presupposing that electric signals are represented by (1), the recognition of disturbance patterns composed of multiple disturbances cannot be an easy task to be accomplished as in the case of single disturbance ocurrence. In fact, the incidence of more than one disturbance in the electric signals can lead to techniques attaining reduced classification performance due to the complexity of classification region if the standard paradigm, which is depicted in Figure 3, is considered. It refers to the fact that in the standard paradigm, the feature vector $\mathbf{p}_{x}$ is extracted directly from the vector $\mathbf{x}=\mathbf{f}+\mathbf{h}+\mathbf{i}+\mathbf{t}_{\mathrm{imp}}+\mathbf{t}_{\mathrm{dec}}+\mathbf{t}_{\mathrm{dam}}+\mathbf{v}$ and the vector $\mathbf{p}_{x}$ can be unfavorable for disturbance classification purpose because the vector $\mathbf{x}$ is composed of several components, which are 


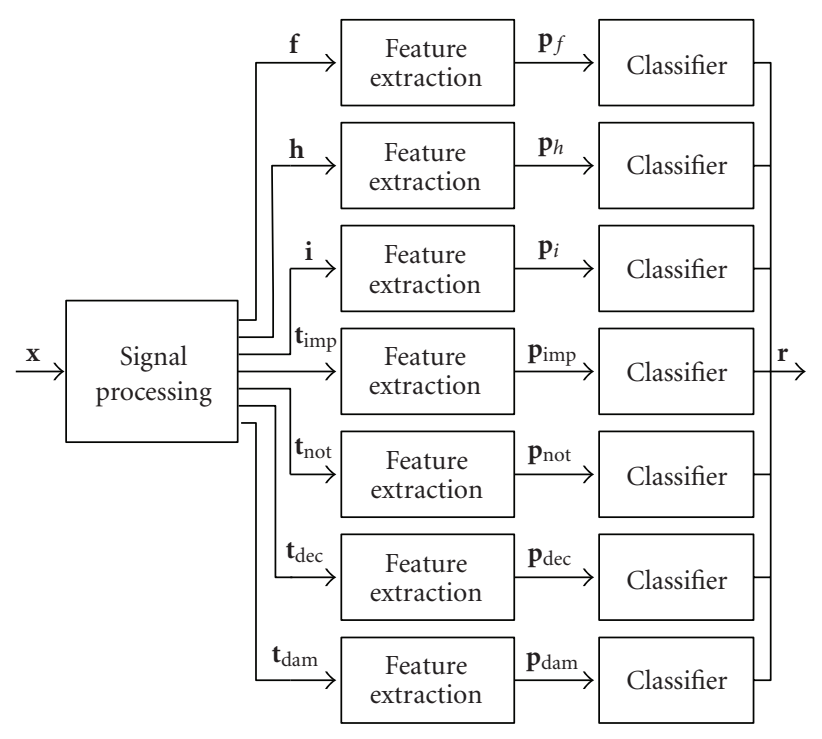

Figure 4: Novel paradigm for the classification of single and multiple disturbances.

associated with disjoint disturbances sets. As a result, the design of pattern recognition technique for classifying multiple disturbances is a very difficult task to be accomplished [5, 7]. One can state that this is true because the electric signals are in the majority of cases composed of complex patterns, which is constituted by multiple primitive patterns. Therefore, the surfaces among the classification regions that are associated with different types of single and multiple disturbances in the feature vector space, which is defined by the set of feature vectors $\mathbf{p}_{x}$, can be very complex and difficult to attain, even though powerful feature extraction and classification techniques are applied. As a result, the design of pattern recognition techniques offer low performance if (1) is composed of multiple disturbances; see $[2,3]$ and reference therein. References [2, 3] are the first contributions proposing pattern recognition techniques to classify one or two simultaneous disturbances in voltage signals. The attained results with synthetic data is lower than $95 \%$, see [2]. These results illustrate that a lot of efforts have to be put in for the development of powerful pattern recognition techniques capable of achieving high performance.

To overcome the weakness and reduced performance of the standard paradigm, in the following a paradigm based on the principle of divide to conquer is presented, which has been widely and succeessfully applied to many engineering applications, to design powerful and efficient disturbance classification techniques for PQ applications. In this paradigm, the vector $\mathbf{x}$ is decomposed into what we call primitive components from which individual disturbances or, as defined here, primitive patterns can be easily classified. Here, primitive components are defined as those components from which only single disturbances can be straightforwardly classified. The primitive components are the vectors separately constituted by samples of signals expressed by (2), (3), (4), (8), (9), (10), and (11). Figure 4 illustrates the whole new paradigm.
As it can be seen, the main idea is to divide the powerline signals into several primitive components in which simple pattern recognition techniques can be designed easily and applied. The motivations for decomposing the vector $\mathbf{x}$ into vectors $\mathbf{f}, \mathbf{h}, \mathbf{i}, \mathbf{t}_{\text {imp }}, \mathbf{f}_{\text {not }}, \mathbf{t}_{\mathrm{dec}}$, and $\mathbf{t}_{\mathrm{dam}}$ are as follows.

(i) From vector $\mathbf{f}$, several disjoint disturbances that are mainly related to the fundamental component can classify easily. For the vector $\mathbf{f}$, the primitive patterns are named sag, swell, interruption, sustained interruption, undervoltage, and overvoltage. As a result, the classification of disturbances in the fundamental component can be formulated as the decision between four hypotheses [33-35]:

$$
\begin{gathered}
\mathscr{H}_{f, 1}: \mathbf{f}=\mathbf{f}_{\text {norm }}+\mathbf{v}_{f}, \\
\mathscr{H}_{f, 2}: \mathbf{f}=\mathbf{f}_{\text {under }}+\mathbf{v}_{f}, \\
\mathscr{H}_{f, 3}: \mathbf{f}=\mathbf{f}_{\text {over }}+\mathbf{v}_{f}, \\
\mathscr{H}_{f, 4}: \mathbf{f}=\mathbf{f}_{\text {inter }}+\mathbf{v}_{f},
\end{gathered}
$$

where $\mathbf{v}_{f}$ is the noise vector associated with the fundamental component. The vectors $\mathbf{f}_{\text {norm }}, \mathbf{f}_{\text {under }}, \mathbf{f}_{\text {over }}$, and $\mathbf{f}_{\text {inter }}$ denote a normal condition of fundamental component, an undervoltage or sag, a disturbance called overvoltage or swell, and a disturbance named sustained interruption or interruption, respectively. One has to note that the hypothesis expressed by (12) can be split into four simple hypotheses which are expressed by

$$
\begin{gathered}
\mathscr{H}_{f, i, 0}: \mathbf{f}=\mathbf{v}_{f}, \\
\mathcal{H}_{f, i, 1}: \mathbf{f}=\mathbf{f}_{\text {dist }}+\mathbf{v}_{f},
\end{gathered}
$$

where dist denotes norm, under, over, and inter if $i=$ $1, \ldots, 4$, respectively.

(ii) From vector $\mathbf{h}$, one can recognize the occurrence of distortions generated by the harmonic sources which mainly are nonlinear loads connected to power systems. Here the primitive pattern is called harmonic distortion. By extracting the vector $\mathbf{h}$ from the vector $\mathbf{x}$, the problem related to classifying the disturbances as harmonic distortion in voltage and current signals can be formulated as follows $[33,34]$ :

$$
\begin{gathered}
\mathscr{H}_{h, 1,0}: \mathbf{h}_{h}=\mathbf{v}_{h}, \\
\mathscr{H}_{h, 1,1}: \mathbf{h}_{h}=\mathbf{h}+\mathbf{v}_{h},
\end{gathered}
$$

where $\mathbf{v}_{h}$ is the noise vector associated with the harmonic components. One can see that this allows the use of simple detection technique to recognize the presence of harmonics.

(iii) The vector $\mathbf{i}$ is related to the incidence of interharmonic components in the electric signals. These components appear due to the occurrences of flicker as well as power electronic-based equipment. Here, the primitive pattern is just called interharmonic. This primitive pattern can be further decomposed into other primitive patterns if one needs to analyze some specific groups of interharmonic components. Note that flicker is a very specific class of interharmonic in which the frequency is in the range $0<f<f_{0}$ [36]. The classification of the interharmonic components in voltage and 
current signals can then be formulated as a decision between two simple hypotheses $[33,34]$ :

$$
\begin{gathered}
\mathscr{H}_{i, 1,0}: \mathbf{i}_{i}=\mathbf{v}_{i}, \\
\mathscr{H}_{i, 1,1}: \mathbf{i}_{i}=\mathbf{i}+\mathbf{v}_{i},
\end{gathered}
$$

where $\mathbf{v}_{i}$ is the noise vector associated with the inter-hamonic components.

(iv) The use of $\mathbf{t}_{\text {imp }}$ vector provides us with the means to detect the occurrence of impulsive transients in the powerline signals. Then, the classification of primitive pattern as impulsive transient in voltage and current signals can be formulated as a decision between two simple hypotheses $[33,34]$ :

$$
\begin{gathered}
\mathscr{H}_{t_{\mathrm{imp}}, 1,0}: \mathbf{t}_{t_{\mathrm{imp}}}=\mathbf{v}_{\mathrm{imp}}, \\
\mathscr{H}_{t_{\mathrm{imp}}, 1,1}: \mathbf{t}_{t_{\text {imp }}}=\mathbf{t}_{\mathrm{imp}}+\mathbf{v}_{\mathrm{imp}},
\end{gathered}
$$

where $\mathbf{v}_{\text {imp }}$ is the noise vector associated with the disturbance named impulsive transient.

(v) The use of $\mathbf{t}_{\text {not }}$ vector allows the identification of primitive pattern called notch in the powerline signals and, consequently, the presence of power electronic devices. Regarding the use of vector $\mathbf{t}_{\text {not }}$, this classification problem can be formulated as a decision between two simple hypotheses $[33,34]$ :

$$
\begin{gathered}
\mathscr{H}_{t_{\mathrm{not}}, 1,0}: \mathbf{t}_{t_{\mathrm{not}}}=\mathbf{v}_{\mathrm{not}}, \\
\mathscr{H}_{t_{\mathrm{not}}, 1,1}: \mathbf{t}_{t_{\mathrm{not}}}=\mathbf{t}_{\mathrm{not}}+\mathbf{v}_{\mathrm{not}},
\end{gathered}
$$

where $\mathbf{v}_{\text {imp }}$ is the noise vector associated with the disturbance called notch.

(vi) The use of $\mathbf{t}_{\mathrm{dec}}$ vector offers a means to recognize the so-called oscillatory transient (primitive pattern) that is defined as sudden, nonpower frequency changes in the steadystate condition of voltage and/or current that include both positive and negative polarity values. By extracting the vector $\mathbf{t}_{\mathrm{dec}}$ from the vector $\mathbf{x}$, the problem related to classifying the disturbances as decaying oscillations in voltage and current signals can be formulated as a decision between two simple hypotheses $[33,34]$ :

$$
\begin{gathered}
\mathscr{H}_{t_{\mathrm{dec}}, 1,0}: \mathbf{t}_{t_{\mathrm{dec}}}=\mathbf{v}_{\mathrm{dec}} \\
\mathscr{H}_{t_{\mathrm{dec}}, 1,1}: \mathbf{t}_{t_{\mathrm{dec}}}=\mathbf{t}_{\mathrm{dec}}+\mathbf{v}_{\mathrm{dec}}
\end{gathered}
$$

where $\mathbf{v}_{\mathrm{dec}}$ is the noise vector associated with the disturbance called decaying oscillation.

(viii) The use of $\mathbf{t}_{\mathrm{dam}}$ vector offers us the means to verify the incidence of the primitive pattern characterized as a sudden, nonpower frequency change in the steady-state condition of voltage, current, or both, that is unidirectional in polarity (primarily either positive or negative). The use of $\mathbf{t}_{\text {dam }}$ allows one to recognize damped exponentials from a decision between two simple hypotheses [33, 34]:

$$
\begin{gathered}
\mathcal{H}_{t_{\mathrm{dam}}, 1,0}: \mathbf{t}_{t_{\mathrm{dam}}}=\mathbf{v}_{\mathrm{dam}}, \\
\mathcal{H}_{t_{\mathrm{dam}}, 1,1}: \mathbf{t}_{t_{\mathrm{dam}}}=\mathbf{t}_{\mathrm{dam}}+\mathbf{v}_{\mathrm{dam}},
\end{gathered}
$$

where $\mathbf{v}_{\text {dam }}$ is the noise vector associated with the disturbance called damped decaying.
From all reasons and motivations stated before, it is clear that improved performance can be attained for the classification of single and multiple disturbances in electric signals, if the electric signals can be decomposed into several primitive components. By using such a very simple and powerful idea, which is named the principle of divide to conquer, the design of a very complex classification technique is broken in several simple ones that can be developed easily. The result derived from this paradigm is very interesting because the incidence of several sets of classes of disturbances can be identified easily. In fact, each of the vectors $\mathbf{f}, \mathbf{h}, \mathbf{i}, \mathbf{t}_{\text {imp }}$, $\mathbf{t}_{\mathrm{not}}, \mathbf{t}_{\mathrm{dec}}$, and $\mathbf{t}_{\mathrm{dam}}$ are related to disjointed classes of disturbances and their recognition in parallel can be performed easily.

From a PQ perspective, the advantages and opportunities offered by this paradigm is very appealing and promising to completely characterize the behavior of electric signals not only for classification purpose, but also for other very demanding issues listed at the beginning of Section 1. To make this strategy successful, one has to develop signal processing techniques capable of decomposing the vector $\mathbf{x}$ into the vectors $\mathbf{f}, \mathbf{h}, \mathbf{i}, \mathbf{t}_{\mathrm{imp}}, \mathbf{f}_{\mathrm{not}}, \mathbf{t}_{\mathrm{dec}}$, and $\mathbf{t}_{\mathrm{dam}}$ to allow the further extraction of simple and powerful feature extraction and the use of simple classifiers.

This is a very hard and difficult problem to be solved so that it should be deeply investigated by signal processing researchers interested in this field. In fact, the decomposition of vector $\mathbf{x}$ into the vectors $\mathbf{f}, \mathbf{h}, \mathbf{i}, \mathbf{t}_{\mathrm{imp}}, \mathbf{t}_{\mathrm{not}}, \mathbf{t}_{\mathrm{dec}}$, and $\mathbf{t}_{\mathrm{dam}}$ is not a simple task to be accomplished with simple signal processing techniques. However, if one assumes that the vector $\mathbf{x}$ is given by

$$
\mathbf{x}=\mathbf{f}+\mathbf{v}_{f}+\mathbf{h}+\mathbf{v}_{h}+\mathbf{u}+\mathbf{v}_{u}
$$

where $\mathbf{v}=\mathbf{v}_{f}+\mathbf{v}_{h}+\mathbf{v}_{u}$ and

$$
\mathbf{u}=\mathbf{i}+\mathbf{t}_{\mathrm{imp}}+\mathbf{t}_{\mathrm{not}}+\mathbf{t}_{\mathrm{dec}}+\mathbf{t}_{\mathrm{dam}}
$$

then some signal processing techniques can be applied to decompose $\mathbf{x}$ into the vectors $\mathbf{f}, \mathbf{h}$, and $\mathbf{u}$. And, as a result, highperformance pattern recognition technique for a limited and very representative set of disturbances in electric signals can be designed. In fact, the decomposition of the vector $\mathbf{x}$ into the vectors $\mathbf{f}, \mathbf{h}$, and $\mathbf{u}$ allows one to design classification techniques for disjoint sets of disturbances associated with the primitive components named fundamental, harmonic, and transient, respectively. Section 3 introduces a pattern recognition technique for single and multiple disturbances that makes use of (20)-(21) and attains an interesting improvement.

\section{PROPOSED TECHNIQUE}

The scheme of the proposed technique is portrayed in Figure 5. Note that in the signal processing block, algorithms responsible for extracting the vectors $\mathbf{f}, \mathbf{h}$, and $\mathbf{u}$ are implemented. 


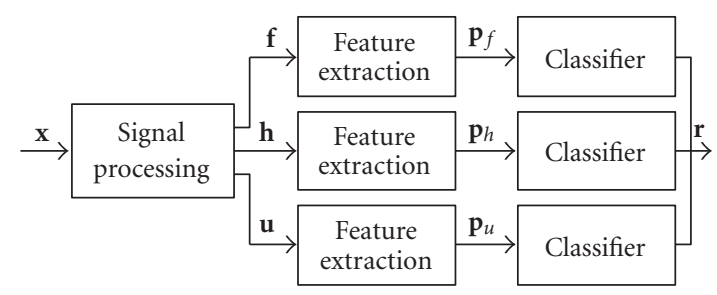

FIgURE 5: Standard paradigm for the classification of single and multiple disturbances.

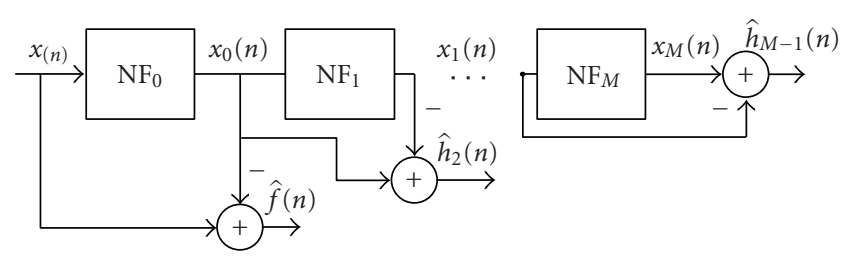

Figure 6: Scheme of the signal processing block.

This signal processing block is illustrated in Figure 6, where the blocks $N F_{i}, i=0, \ldots, M-1$, implement secondorder notch filter with notch frequency $\omega_{m}=2 m \pi\left(f_{0} / f_{s}\right)$. These filters are responsible for the estimations of $\{f(n)\}$, $\{h(n)\}$, and $\{u(n)\}$. The z-transform of the second-order notch filter is expressed by

$$
H_{m}(z)=\frac{1+a_{m} z^{-1}+z^{-2}}{1+\rho_{m} a_{m} z^{-1}+\rho_{m}^{2} z^{-2}},
$$

where

$$
a_{m}=-2 \cos m \omega_{0}
$$

and $0 \ll \rho_{m}<1$ is the notch factor. One should note that the notch filter has some drawbacks regarding the choice of the parameter $\rho_{m}$, and also its output is, by definition, a contribution of information of its own internal state and the input. As a result, the notch filter can produce transient signals that reflect the changes at the input and in its states. This could be a problem if the aim is to estimate the parameters of the primitive components. For this problem, the use of high-order notch filter, such as 4th order or higher ones, can be used to reduce the transient at the output of the notch filter [37]. Although, these transients can contribute to distort the primitive components, we point out that such behavior does not minimize the classification performance. In fact, the transients at the output of the notch filter shows a typical parttern for each disturbance, then a neglible loss of performance has been verified for disturbance detection, see $[1,38]$. An advantage regarding the use of notch filter is that its implementation with finite word length in the $\delta$-operator domain is very robust against the effects of finite precision, then it can be implemented in a cheap digital signal processor
(DSP)-based equipment running with finite-precision. The notch filter in $\delta$-operator domain is given by $[39,40]$

$$
H_{m}(\delta)=\left.H_{m}(z)\right|_{z=1+\Delta \delta}=\frac{1+\alpha_{m, 1} \delta^{-1}+\alpha_{m, 2} \delta^{-2}}{1+\beta_{m, 1} \delta^{-1}+\beta_{m, 2} \delta^{-2}},
$$

where

$$
\begin{aligned}
& \alpha_{m, 1}=\frac{2}{\Delta}\left(1-\cos m \omega_{0}\right), \\
& \alpha_{m, 2}=\frac{2}{\Delta^{2}}\left(1-\cos m \omega_{0}\right), \\
& \beta_{m, 1}=\frac{2}{\Delta}\left(1-\rho_{m} \cos m \omega_{0}\right), \\
& \beta_{m, 2}=\frac{1+\rho_{m}^{2}-2 \rho_{m} \cos \omega_{0}}{\Delta^{2}},
\end{aligned}
$$

where $0<\Delta \ll 1$ is carefully chosen to minimize roundoff error effects. Although the implementation of a filter in the $\delta$ operator domain demands more computational complexity, it is very robust to the quantization effects when the sampling rate is at least 10 times higher than the frequency band of interest.

The vectors $\mathbf{f}, \mathbf{h}$, and $\mathbf{u}$ provided at the processing block output are expressed by

$$
\begin{aligned}
\mathbf{f} & =\widehat{\mathbf{f}}, \\
\mathbf{h} & =\sum_{m=2}^{M} \hat{\mathbf{h}}_{m}, \\
\mathbf{u} & =\mathbf{x}_{M},
\end{aligned}
$$

respectively, where $\mathbf{f}=[\hat{f}(n) \cdots \hat{f}(n-N+1)]^{T}, \widehat{\mathbf{h}}_{m}=$ $\left[\hat{h}_{m}(n) \cdots \hat{h}_{m}(n-N+1)\right]^{T}$, and $\mathbf{x}_{M}=\left[x_{M}(n) \cdots x_{M}(n-\right.$ $N+1)]^{T}$. If we assume $\rho_{m}, m=0,1, \ldots, M$, are very close to a unity, then

$$
x_{i}(n) \cong\left\{\begin{aligned}
& x(n+\left.d_{0}\right)-\left|H_{0}\left(e^{j \omega_{0}(n)}\right)\right| A_{0}(n) \\
& \times \cos \left(n \omega_{0}(n)+\theta_{0}(n)+\Delta \theta_{0}(n)\right) \\
& \text { if } i=0 \\
& x_{i-1}(\left(n+d_{i-1}\right)-\sum_{m=0}^{i}\left|H_{m}\left(e^{j m \omega_{0}(n)}\right)\right| A_{m}(n) \\
& \times \cos \left(n m \omega_{0}(n)+\theta_{m}(n)+\Delta \theta_{m}(n)\right) \\
& \text { otherwise }
\end{aligned}\right.
$$

where

$$
\begin{gathered}
\Delta \theta_{m}(n)=\sum_{k=0}^{i} \angle H_{k}\left(m \omega_{0}(n)\right), \\
\sigma_{m}^{2}(n)=\sigma_{v}^{2}\left(1-\prod_{k=0}^{i}\left|H_{k}\left(m \omega_{0}(n)\right)\right|^{2}\right) .
\end{gathered}
$$

The technique implemented in the feature extraction blocks is responsible for extracting reduced and representative vectors of features $\mathbf{p}_{i}, i=f, h, u$, from the vectors $\mathbf{f}$, $\mathbf{h}$, and $\mathbf{u}$, respectively. Sections 3.1, 3.2, and 3.3 deal with feature extraction, feature selection, and classification techniques that are considered in this contribution. Once the feature vectors $\mathbf{p}_{i}, i=f, h, u$, are extracted, the blocks named 


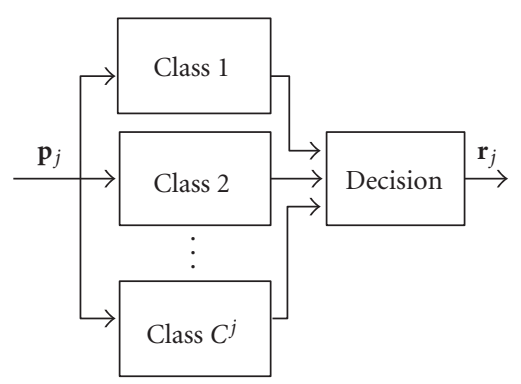

FIGURE 7: Scheme of the classification block.

classifier, which implement the algorithms that decide by the incidence or not of disturbances in the vectors $\mathbf{f}, \mathbf{h}$, and $\mathbf{u}$, are evaluated.

From the vector $\mathbf{f}$, four disjoint patterns of disturbances, which are named sag, swell, normal, and interruption, are primitive patterns. So, the hypothesis test formulated in (13) is applied. If one considers the vector $\mathbf{h}$, then one primitive pattern called harmonic is defined and the hypothesis test formulated in (14) is considered. Finally, for the vector $\mathbf{u}$, it is well known that at least five disturbances or primitive patterns (interharmonics, spikes, notches, decaying oscillations, and damped exponentials) can occur simultaneously in the vector $\mathbf{u}$. As a result, $2^{5}=32$ classes of disturbances can be associated with the vector $\mathbf{u}$ and a very complex hypotheses test should be formulated.

As the primitive patterns are being considered in this work, Figure 7 portrays the scheme of the classification techniques applied in the classifier blocks. Note that each class block makes use of a simple classification technique $i=$ $1, \ldots, C^{j}, j=f, h, u$, that is responsible for classifying each disturbance in the vectors $\mathbf{f}, \mathbf{h}$, and $\mathbf{u}$. Since Figure 7 refers to the classifier block applied to the feature vector $\mathbf{p}_{f}$, then $C^{f}=4 . C^{h}=1$ if the feature vector $\mathbf{p}_{h}$ is being analyzed. Finally, $C^{u}=32$ when one tries to classify the disturbances in the feature vector $\mathbf{p}_{u}$. Regarding $\mathbf{u}$, one has to note that usually three, two, or one disturbances can occur and, consequently, the number of disturbances classes are different for each situation.

While the design of pattern classifiers to work with the feature vectors extracted from vectors $\mathbf{f}$ and $\mathbf{h}$ are quite simple, the design of those techniques for disturbances classification in the vector $\mathbf{u}$ could be a very hard task to be accomplished. However, it is worth stating that the difficulties associated with the proposed scheme are lower than the ones associated with standard techniques such as the ones proposed in $[2,3]$; see results in Section 4. In fact, the proposed technique provides higher performance than the recently developed techniques for single and multiple disturbances.

\subsection{Feature extraction based on high-order-statistics (HOS)}

As stated in [41]: Feature extraction methods determine an appropriate subspace of dimensionality $m$ (either in a linear or a nonlinear way) in the original feature space of dimensionality $d$. Linear transforms, such as principal component analysis, factor analysis, linear discriminant analysis, and projection pursuit have been widely used in pattern recognition for feature extraction and dimensionality reduction.

Despite the good performance achieved by these mentioned feature extraction techniques, it has been recently recognized that higher-order-statistics- (HOS-)based techniques are promising approaches for features extraction if the patterns are modeled as non-Gaussian processes. Analyzing vectors $\mathbf{f}, \mathbf{h}$, and $\mathbf{u}$, one should note that these random vectors are usually modeled as an i.i.d. random processes in which the elements present a non-Gaussian probability mass function (p.m.f.).

The cumulants of higher-order statistics provide much more relevant information from the random processes. Besides that, the cumulants are blind to any kind of Gaussian process, whereas 2 nd-order information is not. Then, cumulant-based signal processing methods can handle colored Gaussian noise automatically, whereas 2nd-order methods may not. Therefore, cumulant-based methods boost signal-to-noise ratio when signals are corrupted by Gaussian measurement noise and can capture more information from the random vectors [42].

Remarkable results regarding detection, classification, and system identification with cumulant-based methods have been reported in [42-45]. Also, a recent investigation of HOS for detection of disturbances in voltage signals reported that the HOS-based features extracted from voltage signals can achieve high detection ratio in a frame as short as $1 / 16$ of one-cycle fundamental component immersed in a noisy environment [38].

By setting the lag $\tau_{i}=\tau, i=1,2,3, \ldots$, the expressions of the diagonal slice of second- , third- , and fourthorder cumulant elements of a zero mean and stationary random vector $\mathbf{z}$, which is assumed to be one of the vectors $\mathbf{f}-E\{\mathbf{f}\}, \mathbf{h}-E\{\mathbf{h}\}$, and $\mathbf{u}-E\{\mathbf{u}\}$, are expressed by [42]

$$
\begin{aligned}
& c_{2, z}(i)=E\{z(n) z(n+i)\}, \\
& c_{3, z}(i)=E\left\{z(n) z^{2}(n+i)\right\}, \\
& c_{4, z}(i)=E\left\{z(n) z^{3}(n+i)\right\}-3 c_{2, z}(i) c_{2, z}(0),
\end{aligned}
$$

respectively, where $i$ is the $i$ th lag. Assuming that $\mathbf{z}$ is an $N$ length vector, the standard approximation of (29)-(31) is expressed by

$$
\begin{aligned}
\hat{c}_{2}(i):= & \frac{2}{N} \sum_{n=0}^{N / 2-1} z(n) z(n+i), \\
\hat{c}_{3}(i):= & \frac{2}{N} \sum_{n=0}^{N / 2-1} z(n) z^{2}(n+i), \\
\hat{c}_{4}(i):= & \frac{2}{N} \sum_{n=0}^{N / 2-1} z(n) z^{3}(n+i) \\
& -\frac{12}{N^{2}} \sum_{n=0}^{N / 2-1} z(n) z(n+i) \sum_{n=0}^{N / 2-1} z^{2}(n),
\end{aligned}
$$

respectively, where $i=0,1,2, \ldots, N / 2-1$. 
Recently, other authors proposed the use of (29)-(31) when $i=0$, whose evaluation is carried out by using the standard approximation provided by (32)-(34), for the classification of two disturbances and the attained results were reported between $98 \%$ and $100 \%$, see [46]. In this technique, a 20th-order (very long and complex) elliptic filter to emulate a notch filter responsible for the extraction of the fundamental component and to allow the disturbance classification on the resulting transient signal is applied. One has to note that 4th- or 6th-order notch filter could provide very good performance without such a huge complexity and delay to remove the fundamental component, see [37].

Additionally, we have verified that the technique introduced in [46] leads to a low classification performance due to the following reasons. (i) If the disturbances are related to the fundamental component, then the transient signal could not be representative to allow the classification of disturbances. Note that the disturbances related to the fundamental component are sags, swells, interruptions, and unbalances. It seems to be one reason for the results to be between $98 \%$ and $100 \%$ and not very close to $100 \%$, as reported in Section 4 . (ii) The authors made use of HOS parameters when $i=0$ without the knowledge of the advantages offered by (29)(31). In fact, from (29)-(31), one can note that there is a large number of HOS features to be extracted for further selection. As a result, the classification for two disturbances in voltage signal proposed in [46] is very limited in the sense that many and more representative features could be extracted. (iii) If the electric signals are composed of multiple disturbances, then the feature vector extracted from the transient signals does not allow well-defined classification regions as the ones provided in [46] for only two disturbances. It fatally contributes to decrease the performance of classification technique applied to other disturbances. (iv) The standard approximation to extract HOS-based features is not appropriate if the frame length is short. As a result, a high sampling rate or a long frame length has to be applied to extract representative HOS-based features. One has to note that these concerns, by no means, disregard the use of the technique proposed in [46] for its intentional application. In fact, we are just pointing out the inadequacy of this technique to analyze the incidence of wide-ranging set of single and multiple disturbances in electric signals.

Due to the limitation of (32)-(34) to estimate the HOSbased features and based on the fact that the electric signals can be seen as cyclic or/and quasicyclic ones, we propose in this contribution the use of this information to define other approximation of HOS parameters. By using this information into (29)-(31), the new approximation for the HOSbased feature extractions can be expressed as follows:

$$
\begin{gathered}
\tilde{c}_{2, z}(i):=\frac{1}{N} \sum_{n=0}^{N-1} z(n) z[\bmod (n+i, N)], \\
\tilde{\mathcal{c}}_{3, z}(i):=\frac{1}{N} \sum_{n=0}^{N-1} z(n) z^{2}[\bmod (n+i, N)],
\end{gathered}
$$

$$
\begin{aligned}
\tilde{c}_{4, z}(i):= & \frac{1}{N} \sum_{n=0}^{N-1} z(n) z^{3}[\bmod (n+i, N)] \\
& -\frac{3}{N^{2}} \sum_{n=0}^{N-1} z(n) z[\bmod (n+i, N)] \sum_{n=0}^{N-1} z^{2}(n),
\end{aligned}
$$

where $i=0,1,2, \ldots, N-1$ and $\bmod (a, b)$ is the modulus operator, which is defined as the remainder obtained from dividing $a$ by $b$. The approximations presented in (35)(37) lead to a very interesting result where one has a shortened finite-length vector from which HOS-based parameters have to be extracted. The use of $\bmod (\cdot)$ operator means that we are assuming that the vector $\mathbf{z}$ is an $N$-length cyclic vector. The reason for this refers to the fact that by using such very simple assumption we can evaluate the approximation of HOS-based parameters with all available $N$ samples. Therefore, a reduced sampling rate and/or a shortened frame length could be valuable for HOS parameters estimation. That is one of the reasons for the improved performance achieved by the proposed technique in Section 4 . The use of (35)-(37) for improved disturbance detection was presented in [38].

Now, suppose that the elements of the vector $\mathbf{z}=$ $[z(0), z(1), \ldots, z(N-1)]^{T}$ are organized from the smallest to the largest values and the vector composed of these values are expressed by $\mathbf{z}_{\mathrm{or}}=\left[z_{\mathrm{or}}(0), z_{\mathrm{or}}(1), \ldots, z_{\mathrm{or}}(N-1)\right]^{T}$, where $z_{\text {or }}(0) \leq z_{\text {or }}(1) \leq, \ldots, \leq z_{\text {or }}(N-1)$. If one replaces the vector $\mathbf{z}$ by the vector $\mathbf{z}_{\mathrm{or}}$ in $(32)-(37)$, then the extracted cumulants are named ordered HOS-based features [47]. By doing so, the set of HOS-based features is composed of several elements. The HOS-based feature vector, whose elements are candidates for use in the proposed classification technique, extracted from the vectors $\mathbf{z}$ and $\mathbf{z}_{\mathrm{or}}$, is given by

$$
\mathbf{p}_{i}=\left[\overline{\mathbf{c}}_{z}^{T} \overline{\mathbf{c}}_{z_{\mathrm{or}}}^{T}\right]^{T}, \quad i=1,2,
$$

where $\mathbf{z}$ denotes $\mathbf{f}, \mathbf{h}$, and $\mathbf{u}, i=1$ refers to a normal condition of voltage signals, $i=2$ denotes the incidence of single or multiple disturbances in the vector $\mathbf{z}$,

$$
\begin{aligned}
\overline{\mathbf{c}}_{z} & =\left[\hat{\mathbf{c}}_{z}^{T} \widetilde{\mathbf{c}}_{z}^{T}\right]^{T}=\left[\hat{\mathbf{c}}_{2, z}^{T} \hat{\mathbf{c}}_{3, z}^{T} \hat{\mathbf{c}}_{4, z}^{T} \widetilde{\mathbf{c}}_{2, z}^{T} \widetilde{\mathbf{c}}_{4, z}^{T} \tilde{\mathbf{c}}_{4, z}^{T}\right]^{T}, \\
\overline{\mathbf{c}}_{z_{\mathrm{or}}} & =\left[\hat{\mathbf{c}}_{z_{\mathrm{or}}}^{T} \widetilde{\mathbf{c}}_{z_{\mathrm{or}}}^{T}\right]^{T}=\left[\hat{\mathbf{c}}_{2, z_{\mathrm{or}}}^{T} \hat{\mathbf{c}}_{3, z_{\mathrm{or}}}^{T} \hat{\mathbf{c}}_{4, z_{\mathrm{or}}}^{T} \widetilde{\mathbf{c}}_{2, z_{\mathrm{or}}}^{T} \widetilde{\mathbf{c}}_{3, z_{\mathrm{or}}}^{T} \widetilde{\mathbf{c}}_{4, z_{\mathrm{or}}}^{T}\right]^{T},
\end{aligned}
$$

where

$$
\begin{aligned}
\hat{\mathbf{c}}_{j, z} & =\left[\hat{c}_{j, z}(0) \hat{c}_{j, z}(1) \cdots \hat{c}_{j, z}\left(\frac{N}{2}-1\right)\right]^{T}, \\
\tilde{\mathbf{c}}_{j, z} & =\left[\tilde{c}_{j, z}(0) \tilde{c}_{j, z}(1) \cdots \tilde{c}_{j, z}(N-1)\right]^{T}, \\
\hat{\boldsymbol{c}}_{j, z_{\mathrm{or}}} & =\left[\hat{c}_{j, z_{\mathrm{or}}}(0) \hat{c}_{j, z_{\mathrm{or}}}(1) \cdots \hat{c}_{j, z_{\mathrm{or}}}\left(\frac{N}{2}-1\right)\right]^{T}, \\
\tilde{\mathbf{c}}_{j, z_{\mathrm{or}}} & =\left[\tilde{c}_{j, z_{\mathrm{or}}}(0) \tilde{c}_{j, z_{\mathrm{or}}}(1) \cdots \tilde{c}_{j, z_{\mathrm{or}}}(N-1)\right]^{T},
\end{aligned}
$$

where $j=2,3,4$. 


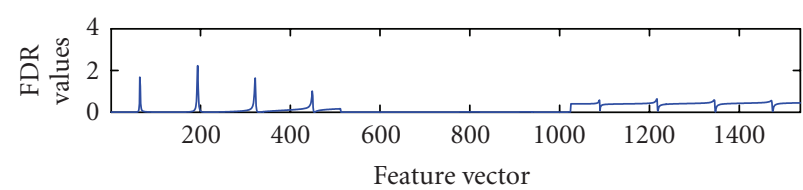

(a)

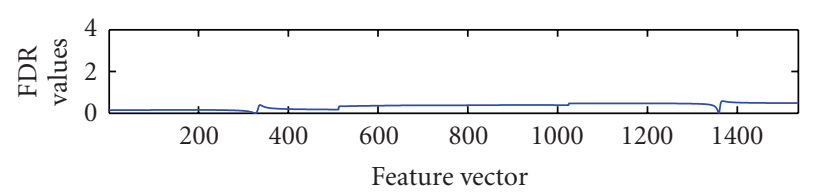

(b)

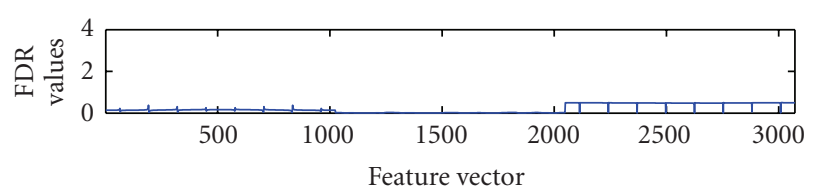

(c)

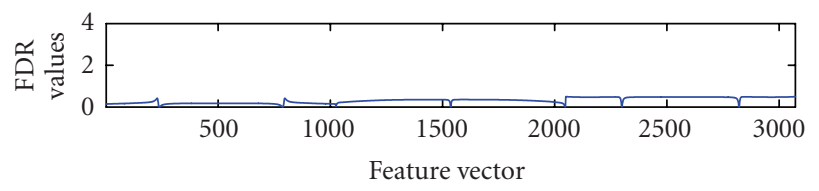

(d)

FIgURE 8: FDR values related to (a) $\hat{\mathbf{c}}_{f}$, (b) $\hat{\mathbf{c}}_{f \text { or }}$, (c) $\tilde{\mathbf{c}}_{f}$, and (d) $\tilde{\mathbf{c}}_{f \text { or }}$ feature vectors when the disturbance is sag.

\subsection{Feature selection technique}

As commented in [41] "The problem of feature selection is defined as follows: given a set of $d$ features, select a subset of size $m$ that leads to the smallest classification error. The feature selection is typically done in an off-line manner and the execution time of a particular algorithm is not as critical as the optimality of the feature subset it generates."

The need for the use of feature selection technique in the set of features extracted from voltage and current signals is due to the fact that the feature set is very large. Aiming at the choice of a representative, finite, and reduced set of features from powerline signals that provides a good separability among distinct classification regions associated with all primitive patterns, the use of the Fisher's discriminant ratio (FDR) is applied [48].

The reason for using the FDR and not other feature selection technique such as sequential forward floating search (SFFS) or sequential backward floating search (SBFS) is that the FDR technique presented good results for this application. The FDR vector which leads to a separability in a lowdimensional space between sets of feature vectors associated

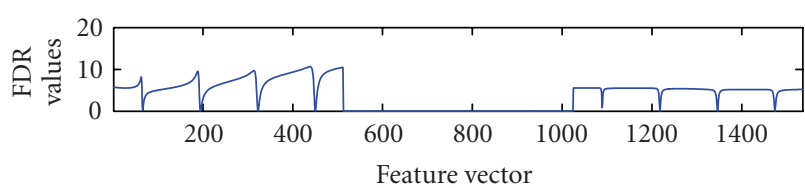

(a)

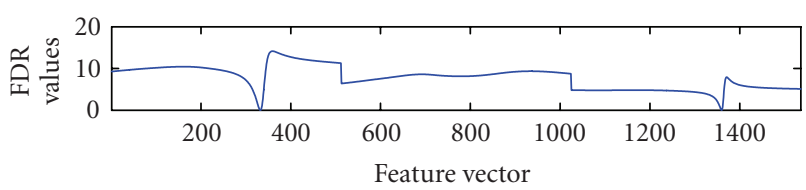

(b)

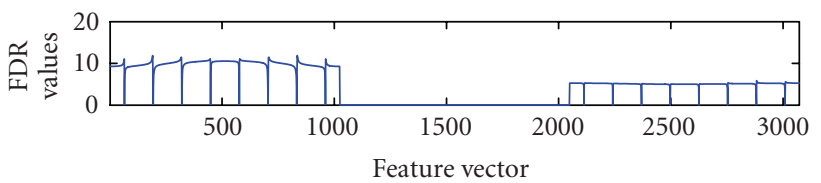

(c)

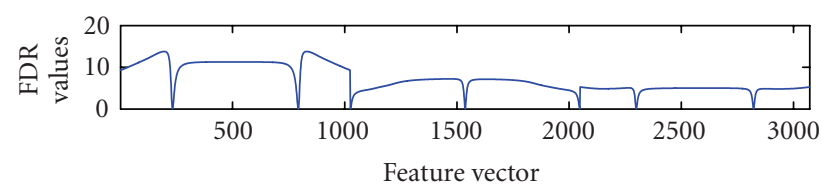

(d)

FIgURE 9: FDR values related to (a) $\hat{\mathbf{c}}_{f}$, (b) $\hat{\mathbf{c}}_{f \text { or }}$, (c) $\tilde{\mathbf{c}}_{f}$, and (d) $\tilde{\mathbf{c}}_{f_{\text {or }}}$ feature vectors when the disturbance is swell.

with different primitive patterns is given by

$$
\mathbf{J}_{c}=\left(\mathbf{m}_{1}-\mathbf{m}_{2}\right)^{2} \odot \frac{1}{\mathbf{D}_{1}^{2}+\mathbf{D}_{2}^{2}},
$$

where $\mathbf{J}_{c}=\left[J_{1} \cdots J_{L_{l}}\right]^{T}, L_{l}$ is the total number of features, $\mathbf{m}_{1}$ and $\mathbf{m}_{2}$, and $\mathbf{D}_{1}^{2}$ and $\mathbf{D}_{2}^{2}$ are the means and variances vectors of parameters vectors $\mathbf{p}_{1, k}, k=1,2, \ldots, M_{p}$, and $\mathbf{p}_{2, k}$, $k=1,2, \ldots, M_{p} . \mathbf{p}_{1, k}$ and $\mathbf{p}_{2, k}$ are feature vectors extracted from the $k$ th voltage signals with and without disturbances and $M_{p}$ denotes the total number of feature vectors for the classes of disturbances associated with the presence or not of disturbances. The symbol $\odot$ refers to the Hadarmard product $\mathbf{r} \odot \mathbf{s}=\left[r_{0} s_{0} \cdots r_{L_{r}-1} s_{L_{r}-1}\right]^{T}$. The $i$ th element of the FDR vector, see (42), having the highest value, $\mathbf{J}_{c}(i)$, is selected for use in the classification technique. Applying the same procedure, $K$ features associated with the $K$ highest FDR values are selected.

Figures $8,9,10,11,12,13,14$ depict the FDR values for the features extracted from vectors $\mathbf{f}, \mathbf{h}$, and $\mathbf{u}$, respectively, when $N=1024$ and $f_{s}=256 \times 60 \mathrm{~Hz}$. One can note that the large number of extracted feature allows a better choice of features for single and multiple disturbances classification. 


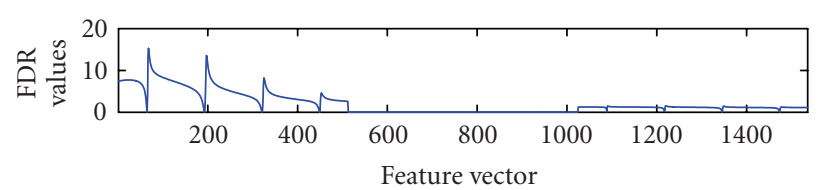

(a)

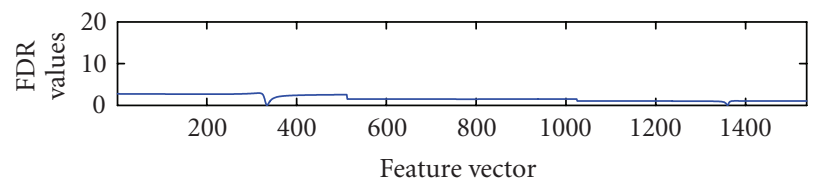

(b)

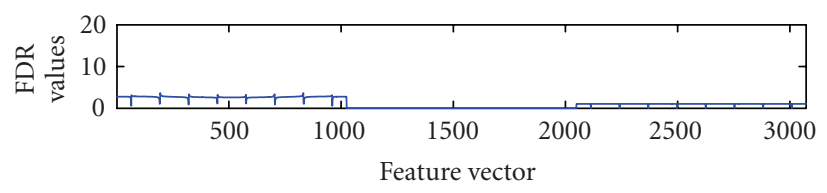

(c)

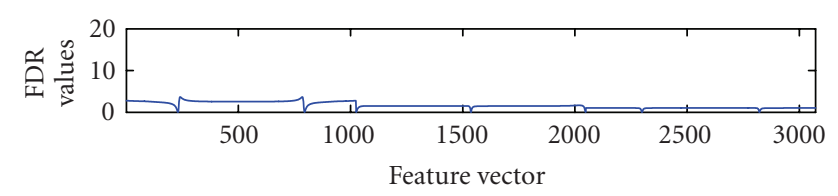

(d)

FIgURE 10: FDR values related to (a) $\hat{\mathbf{c}}_{f}$, (b) $\hat{\mathbf{c}}_{f \text { or }}$, (c) $\tilde{\mathbf{c}}_{f}$, and (d) $\tilde{\mathbf{c}}_{f \text { or }}$ feature vectors when the disturbance is interruption.

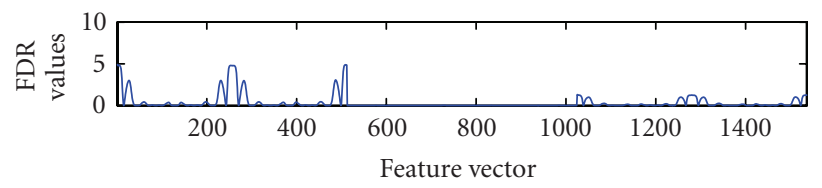

(a)

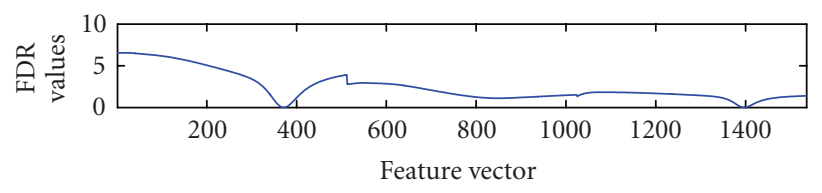

(b)

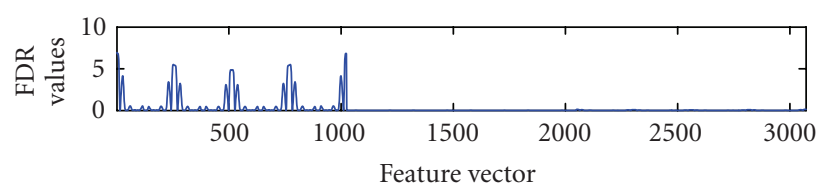

(c)

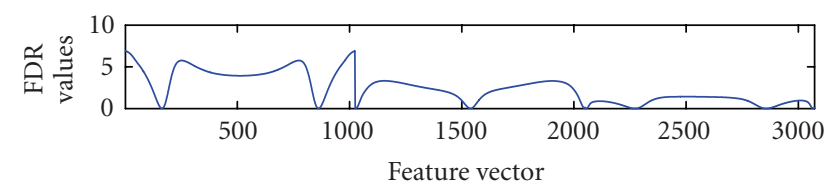

(d)

FIGURE 11: FDR values related to (a) $\hat{\mathbf{c}}_{h}$, (b) $\hat{\mathbf{c}}_{h_{\mathrm{or}}}$, (c) $\tilde{\mathbf{c}}_{h}$, and (d) $\widetilde{\mathbf{c}}_{h_{\mathrm{or}}}$ feature vectors when the disturbance is harmonic.

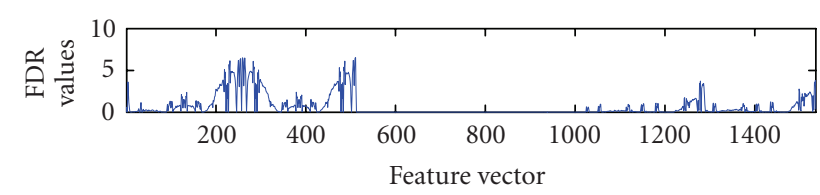

(a)

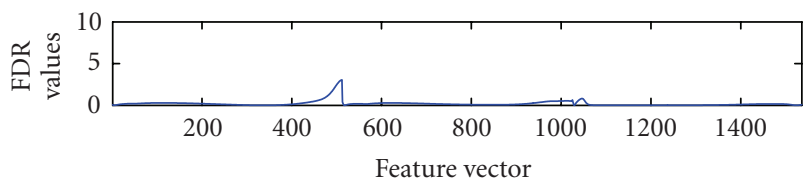

(b)

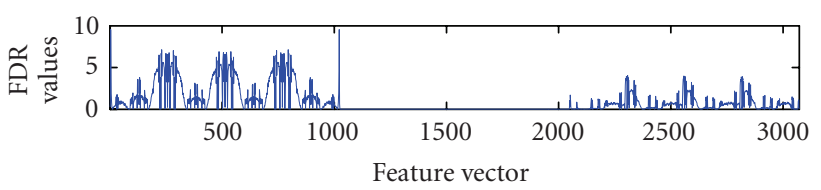

(c)

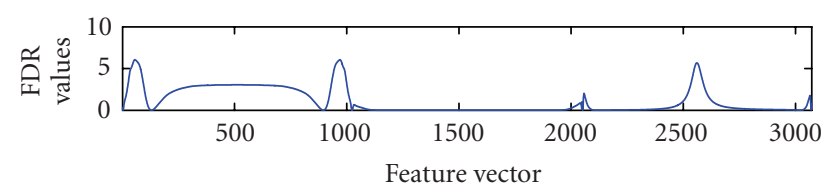

(d)

FIGURE 12: FDR values related to (a) $\hat{\mathbf{c}}_{u}$, (b) $\hat{\mathbf{c}}_{u_{\mathrm{or}}}$, (c) $\tilde{\mathbf{c}}_{u}$, and (d) $\tilde{\mathbf{c}}_{u_{\mathrm{or}}}$ feature vectors when the disturbance is impulsive transient.

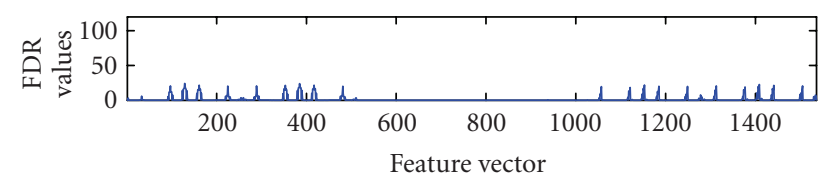

(a)

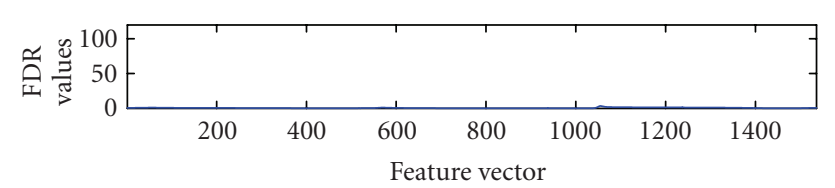

(b)

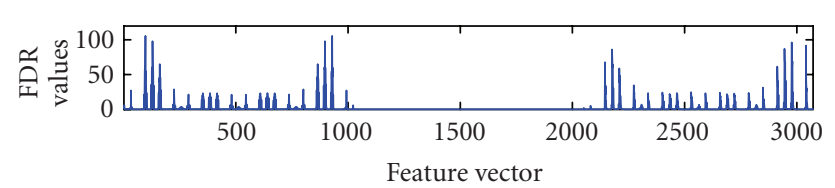

(c)

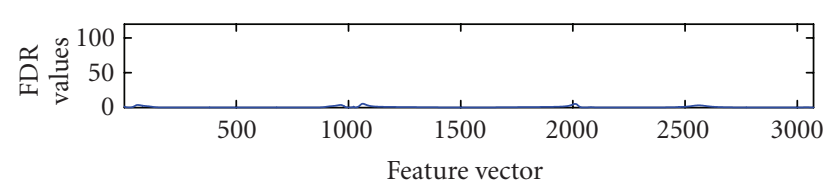

(d)

FIGURE 13: FDR values related to (a) $\hat{\mathbf{c}}_{u}$, (b) $\hat{\mathbf{c}}_{u_{\mathrm{or}}}$, (c) $\tilde{\mathbf{c}}_{u}$, and (d) $\tilde{\mathbf{c}}_{u_{\mathrm{or}}}$ feature vectors when the disturbance is notch. 


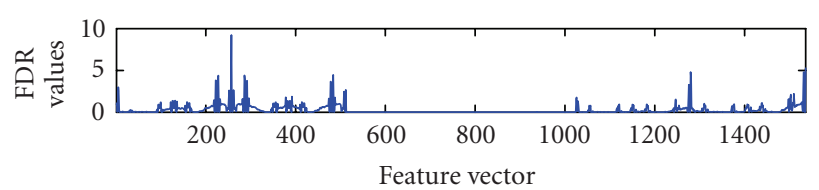

(a)

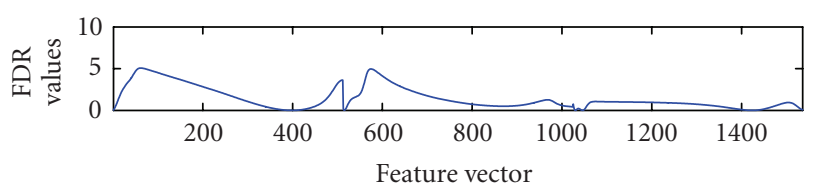

(b)

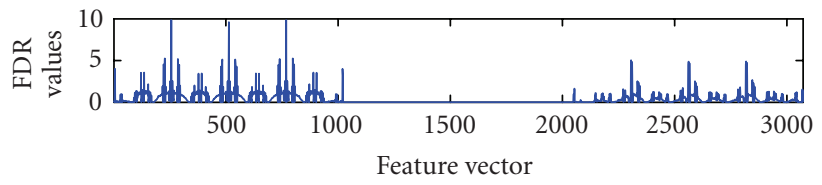

(c)

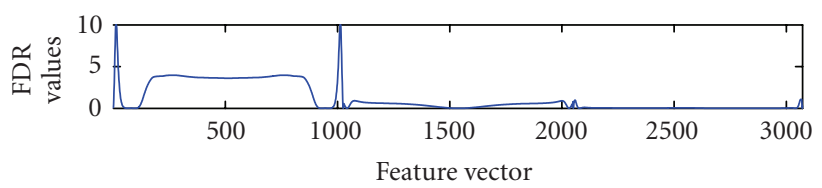

(d)

FIGURE 14: FDR values related to (a) $\widehat{\mathbf{c}}_{u}$, (b) $\widehat{\mathbf{c}}_{u_{\mathrm{or}}}$, (c) $\tilde{\mathbf{c}}_{u}$, and (d) $\widetilde{\mathbf{c}}_{u_{\mathrm{or}}}$ feature vectors when the disturbance is oscillatory transient.

These pictures illustrate very well the limitation of the HOSbased technique proposed in [46]. In fact, the use of HOSbased features when the lag $\tau=0$ is not a good choice because for each primitive pattern there is a distinct set of features that improves the classification ratio. Finally, to evaluate the HOS-based features one can note that for the majority of the disturbance considered, the use of the approximation proposed in this contribution is better than the standard approximation.

\subsection{Pattern recognition technique}

Pattern recognition techniques have been successfully applied to power-quality applications. In these applications, the primitive patterns are represented by feature vector $\mathbf{p}_{j}$, $j=f, h, u$, or attributes viewed as a $d$-dimensional vectors. From the feature vectors, a decision, making process can be summarized as follows: a given pattern is to be assigned to one of $c$ categories $\omega_{1}, \omega_{2}, \omega_{3}, \ldots, \omega_{c}$ based on a vector of $d$ features.

Assuming that the features have a probability of mass function conditioned on the pattern class, then a vector $\mathbf{p}_{j}$, $j=f, h, u$, associated with a pattern belonging to the class $\omega_{i}$ is viewed as an observation drawn randomly from the classconditional probability function $p\left(\mathbf{p}_{j} \mid \omega_{i}\right)$. If the a priori

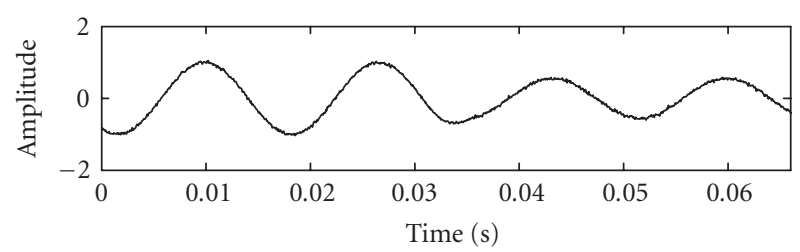

(a)

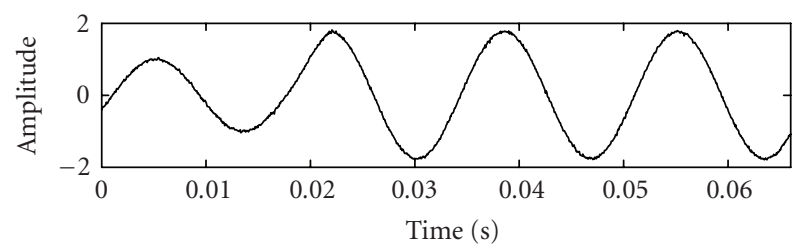

(b)

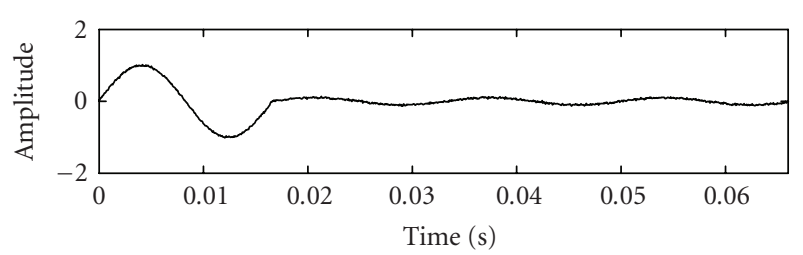

(c)

FIGURE 15: Typical single disturbances related to vector $\mathbf{f}$. (a)-(c) are samples of disturbances called sag, swell, and interruptions, respectively.

probability as well as the parameters of the conditioned probabilities are known, partially known or unknown, then different critera based on Bayes theory (ML, MAP, etc.), NeymanPearson, and so forth can be designed for obtaining the optimum decision boundaries among the $c$ classes in the $d$ dimensional feature vector space.

Assuming that the conditional probabilities functions are known and modeled as Gaussian ones, classifiers based on the maximum likelihood (ML) criterion are designed. If the Gaussian function parameters are estimated from the training data, which is our situation, then the likelihood ratio test of the maximum likelihood (ML) criterion is given by

$$
\frac{p_{\mathbf{P}_{i} \mid \mathscr{H}_{i, j, 0}}\left(\mathbf{p}_{i} \mid \mathscr{H}_{i, j, 0}\right)}{p_{\mathbf{P}_{i} \mid \mathscr{H}_{i, j, 1}}\left(\mathbf{p}_{i} \mid \mathscr{H}_{i, j, 1}\right)}<\frac{\pi_{0}}{\pi_{1}}
$$

where $\pi_{0}=1 / 2$ and $\pi_{1}=1 / 2$ are the a priori probabilities of incidence or not of a single disturbance associated with the voltage signals, $i=f, h, u$ refers to the primitive component represented by the vectors $\mathbf{f}, \mathbf{h}$, and $\mathbf{u} . j=1, \ldots, C^{i}$ is the $j$ th primitive disturbance or primitive pattern associated with the $i$ th primitive component. $\mathbf{p}_{i}, i=f, h, u$, is the feature vector, $\mathscr{H}_{i, j, 0}, j=1, \ldots, C^{i}$, is the hypothesis without 


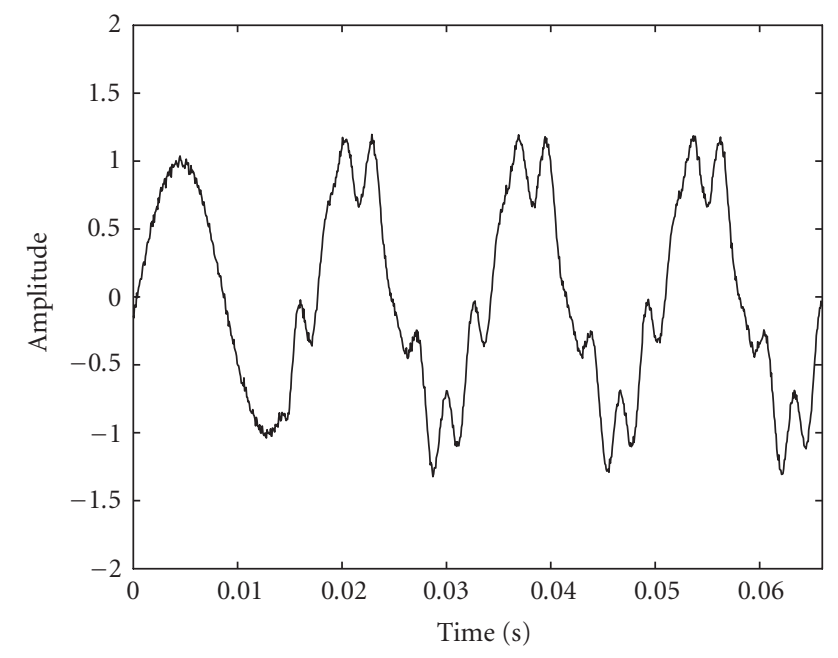

Figure 16: Harmonic disturbance associated with vector $\mathbf{h}$.

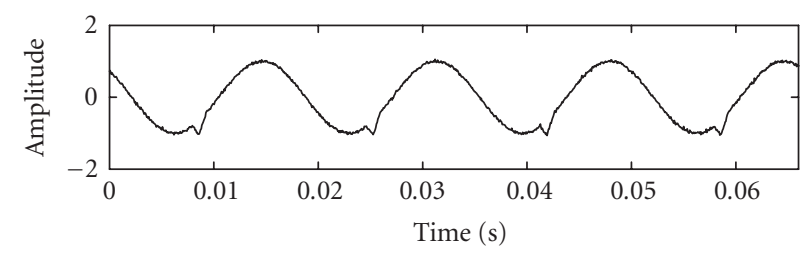

(a)

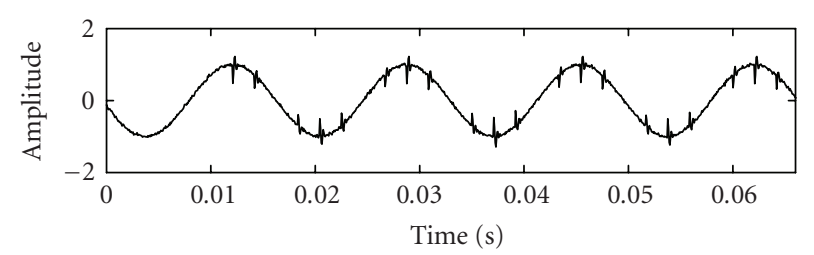

(b)

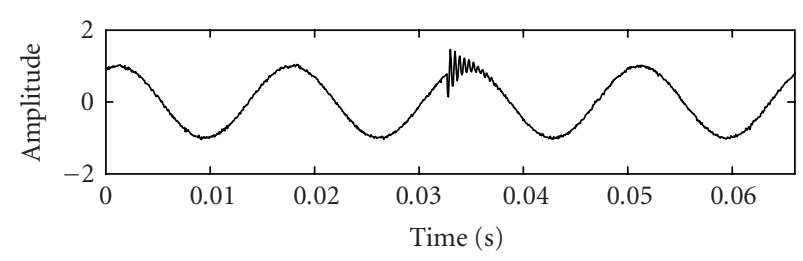

(c)

FIGURE 17: Disturbances associated with the vector $\mathbf{u}$. (a) is a sample of impulsive transient, (b) is a sample of notch, and (c) is a sample of a damped oscillation.

no incidence of the $j$ th primitive disturbance (pattern) in the $i$ th primitive component. $\mathscr{H}_{i, j, 1}, j=1, \ldots, C^{i}$, is the hypothesis with the presence of the $j$ th primitive disturbance in the $i$ th primitive component. Equation (43) is applied to detect the existence of disturbances in the vectors $\mathbf{f}, \mathbf{h}$, and $\mathbf{u}$.

\section{NUMERICAL RESULTS}

The performance of the proposed technique to classify single and multiple disturbances in voltage signals is evaluated and compared with another technique. In Section 4.1, some results obtained with the proposed technique applied to classify single and multiple disturbances in $\mathbf{f}, \mathbf{h}$, and $\mathbf{u}$ components are provided and discussed. Thereafter, in Section 4.2, comparison results between the proposed technique and the one proposed in [2], developed recently to classify single and multiple disturbances in voltage signal by considering the voltage disturbances defined in $[2,3]$, are presented.

\subsection{Performance of the proposed method}

To verify the performance of the proposed technique to classify disturbances in the vectors $\mathbf{f}, \mathbf{h}$, and $\mathbf{u}$, simulations were carried out with several waveforms of voltage signals generated with a sampling rate equal to $f_{s}=256 \times 60$ samples per second (sps) and 32 bits for amplitude quantization. The selected primitive patterns are sag, swell, interruption, harmonic, impulsive transient, notch, and damped oscillation. Figures 15, 16, 17 show single disturbances associated with the vectors $\mathbf{f}, \mathbf{h}$, and $\mathbf{u}$, respectively.

Table 1 lists the attained classification ratio with the proposed technique applied to classify the set of primitive patterns (sag, swell, and interruption) that are associated with the vector $\mathbf{f}$. The following considerations were taken into account for the following simulations: (i) $N=$ $128,256,512,768$, and 1024 , then the lengths of the vector $\mathbf{f}$ correspond to $1 / 2,1,2,3$, and 4 cycles of the fundamental component; (ii) feature vector $\mathbf{p}_{f}$ with four HOSbased features, which were previously selected with the FDR technique, for each primitive pattern; (iii) two thousand sets of primitive patterns generated and equally divided into training and test data; (iv) the use of Bayes detection technique based on the ML criterion as given by (43); (v) $\sigma_{v}^{2}=$ $-30 \mathrm{~dB}$; (vi) the amplitude of the fundamental component, $A_{0}$, assumes values to characterize the disturbances in accordance with the IEEE 1159-1995 Standard [49] and the phase, $\theta_{0}$, is modeled as uniform random variable in the interval $(0,2 \pi]$; (viii) the preprocessing block was implemented with finite word length with 16 bits. Figures 18, 19, 20 portray the selected features extracted from vector f by using the FDR to verify the occurrence of interruptions, sags, and swell. In these plots, the symbols $*$ and $\circ$ are associated with the occurrence or not of each disturbance in the test data. One can note that there are linear separations, then the Bayesian classifiers reduces to a linear ones.

From the results listed in Table 1, one notes that the proposed technique achieves good performance. In Table 1, C means cycles of the fundamental component and CR refers to the classification ratio in percentage. The improvements shown here refer to the attained $\mathrm{CR} \cong 100 \%$ if the lengths of vector $\mathbf{f}$ correspond to $1,2,3$, and 4 cycles of the fundamental component. In the previously developed methods, the results 
TABLe 1: Performance of the proposed technique to classify disturbances in the vector $\mathbf{f}$.

\begin{tabular}{c|ccc}
\hline Frame length & Interruption $(\mathrm{CR}$ in \%) & Sag $(\mathrm{CR}$ in \%) & Swell(CR in \%) \\
\hline $1 C$ & 99.93 & 99.95 & 100 \\
$2 C$ & 99.96 & 99.97 & 100 \\
$3 C$ & 100 & 99.98 & 100 \\
$4 C$ & 100 & 100 & 100 \\
\hline
\end{tabular}

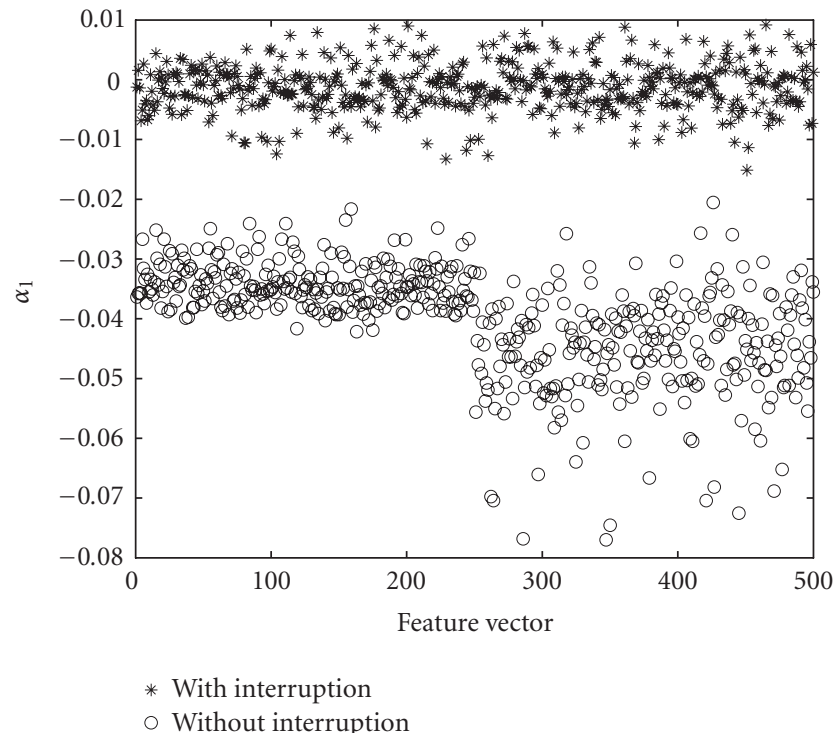

FIGURE 18: HOS-based features extracted from $\mathbf{f}$ for interruption $\alpha_{1}=\hat{c}_{2, f}(68)$.

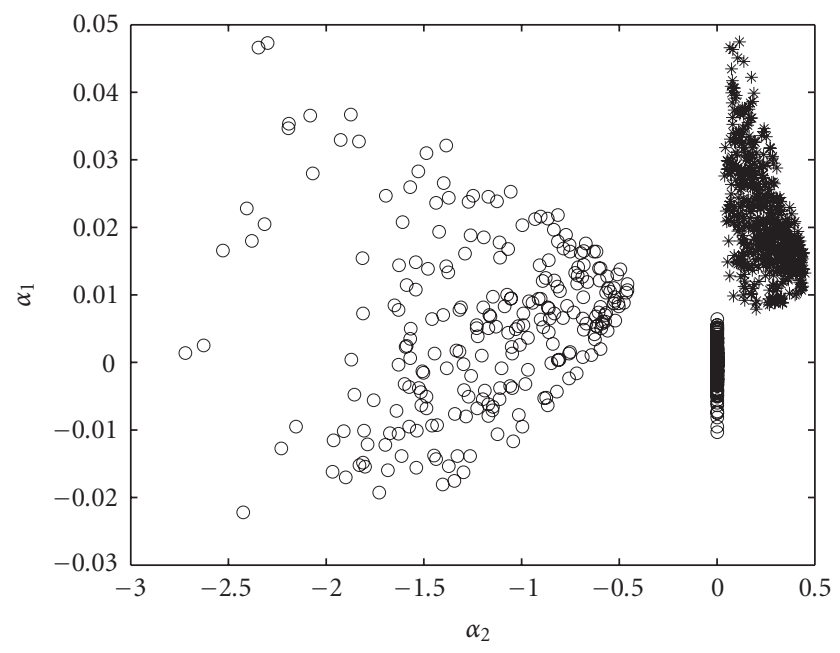

* With sag

$\circ$ Without sag

FIGURE 19: HOS-based features extracted from vector $\mathbf{f}$ for $\operatorname{sag} \alpha_{1}=$ $\tilde{c}_{4, f}(512)$ and $\alpha_{2}=\hat{c}_{2, f}(194)$.

are reported when $N \geq 1024$ or, at least, 4 cycles of the fundamental component, see $[3,5,28,32]$.

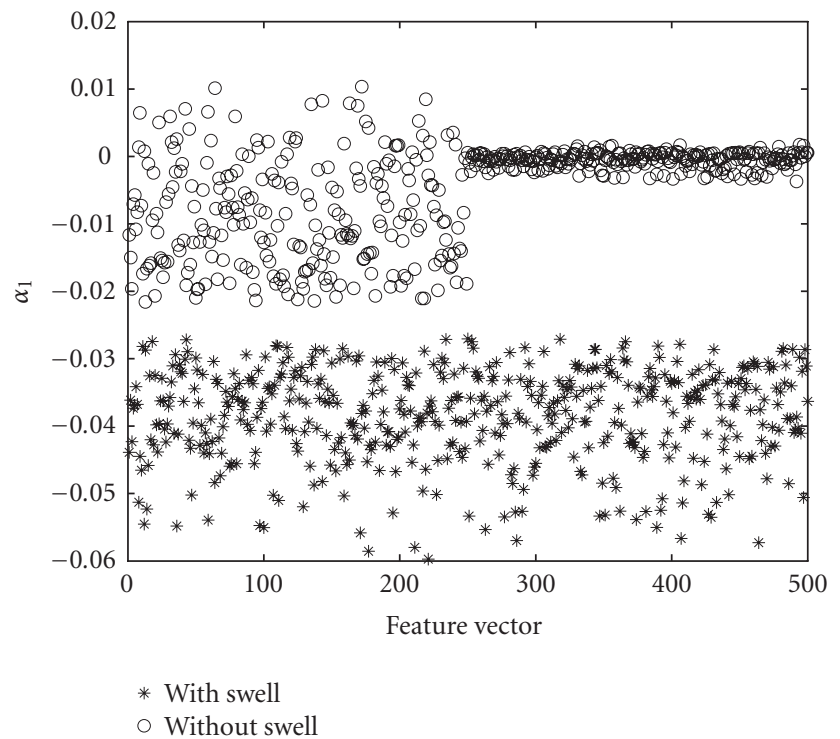

FIGURE 20: HOS-based features extracted from vector $\mathbf{f}$ for swell $\alpha_{1}=\widetilde{c}_{2, f}(191)$.

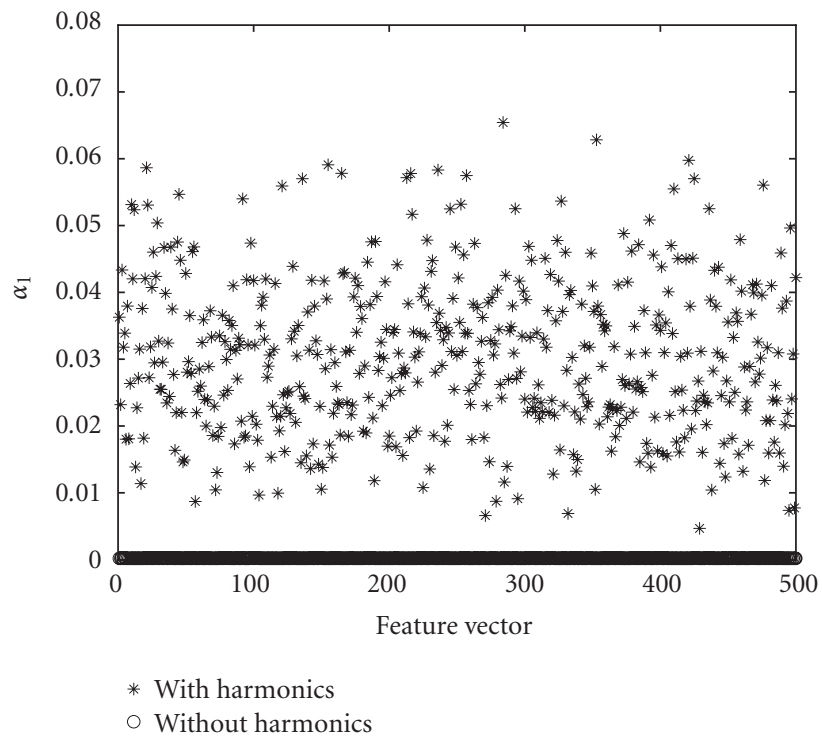

Figure 21: HOS-based features extracted from $\mathbf{h} \alpha=\hat{c}_{2, h}(0)$.

The numerical analysis of the proposed technique for the classification of primitive pattern in voltage signals named 
TABle 2: Performance of the proposed technique for harmonic classification.

\begin{tabular}{c|c}
\hline Frame length & CR in $\%$ \\
\hline $1 / 2 C$ & 100 \\
$1 C$ & 100 \\
$2 C$ & 100 \\
$3 C$ & 100 \\
$4 C$ & 100 \\
\hline
\end{tabular}

harmonic was carried out by taking into account the following considerations: (i) $N=128,256,512,768$, and 1024 and, consequently, the lengths of the vector $\mathbf{h}$ correspond to $1,2,3$, and 4 cycles of the fundamental component; (ii) two thousand sets of harmonic disturbances equally divided in training and test patterns were considered; (iii) Bayes detection technique based on the ML criterion was designed; (iv) $\sigma_{v}^{2}=-30 \mathrm{~dB}$; (iv) the harmonic components, whose frequency are $m \omega_{0}, m=3,5,7,9,11,13$, and 15, have amplitudes $A_{i}$ and $\theta_{i}$ modeled as uniform random variables in the intervals $(0.01,0.4]$ and $(0,2 \pi]$, respectively; (v) feature vector $\mathbf{p}_{h}$ composed of one element selected by FDR criterion. The generated harmonics comply with the IEEE 1159-1995 standard [49]. Figure 21 shows the selected HOS feature obtained from vector $\mathbf{h}$ to detect harmonic presence. The symbols $*$ and $\circ$ are associated with the occurrence or not of harmonic in the test data, respectively.

The results achieved with the proposed technique are presented in Table 2. The CR as high as 100\% is not novel if $N \geq 1024$ or, at least, 4 cycles of the fundamental component are taken into account $[3,5,28,32]$. The novelty here is the fact that the proposed technique is capable of achieving CR as high as $100 \%$ if the length of vector $\mathbf{h}$ corresponds to $1 / 2,1,2,3$, and 4 cycles of the fundamental component.

To check the effectiveness of the proposed technique for the classification of primitive patterns in the vector $\mathbf{u}$, the following considerations were taken into account: (i) $N=1024$, which corresponds to four cycles of the fundamental component; (ii) four-length feature vector $\mathbf{p}_{u}$ in which all elements are selected with the FDR technique; (iii) 3000 sets of disturbances such as impulsive transients, notches, and damped oscillations equally divided into training and test data; (iv) Bayes detection technique based on the ML criterion; (v) additive noise power equal to $\sigma_{v}^{2}=-30 \mathrm{~dB}$; and (vi) single incidence of disturbances in the vector $\mathbf{x}$ that appears in the primitive component represented by the vector $\mathbf{u}$. Figures 22 , 23, 24 depict the selected HOS-based features that were extracted from vector $\mathbf{u}$ to classify the disturbances as oscillatory transient (damped oscillations), impulsive transient, and notch. In these figures, the symbols $*$ and $\circ$ are associated with the occurrence or not of each disturbance in the test data.

The attained results with the test data are shown in Table 3. One can note that the proposed technqiue is capa-

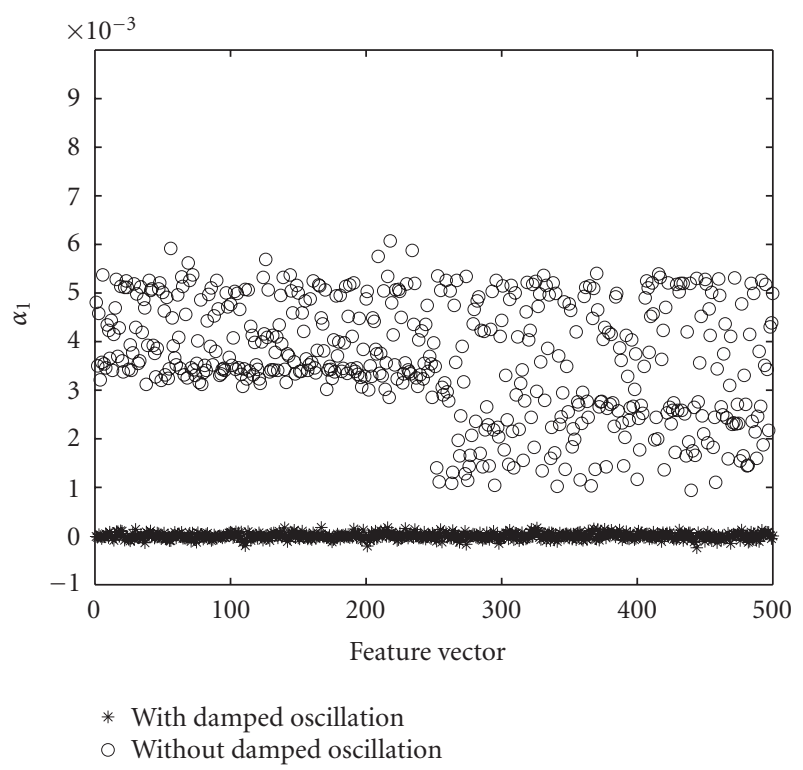

FIgURE 22: HOS-based features extracted from $\mathbf{u}$ to classify the oscillatory transient named damped oscillation, $\alpha_{1}=\widetilde{c}_{2, u}(257)$.

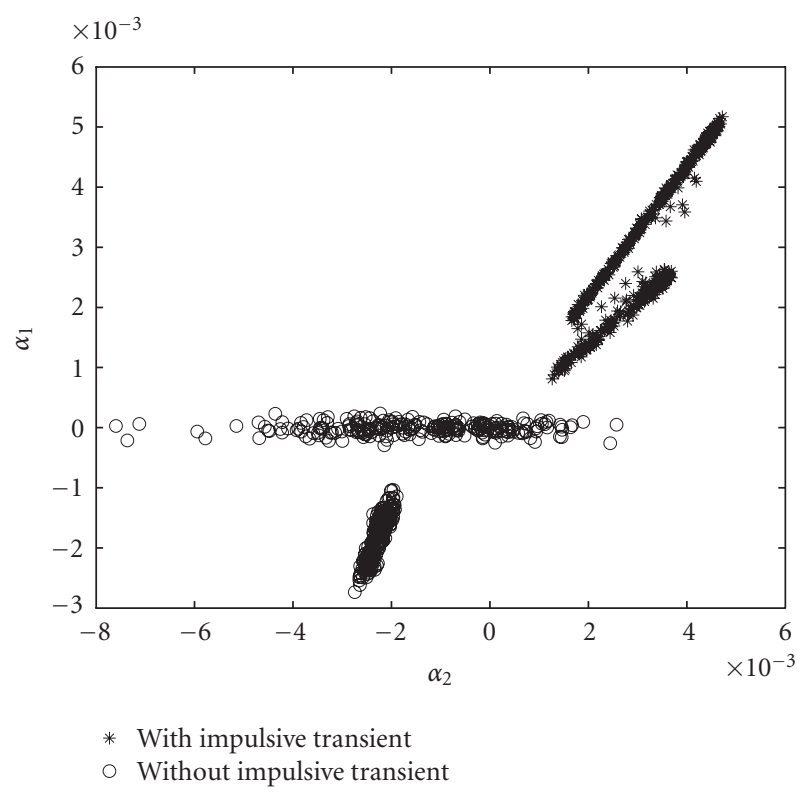

FIGURE 23: HOS-based features extracted from $\mathbf{u}$ to classify the impulsive transient $\alpha_{1}=\widetilde{c}_{2, u}(4)$ and $\alpha_{2}=\widehat{c}_{2, u}(511)$.

ble of classifying almost all primitive patterns in the vector u.

From the results reported in Tables $1-3$, in Figures 8 14, and in Figures 18-24, one can make the following observations: (i) based on the results obtained with FDR feature selection technique, comulants related to 2nd-order statistics are more appropriate features for disturbance classification in voltage signals; (ii) in the majority of the cases, 


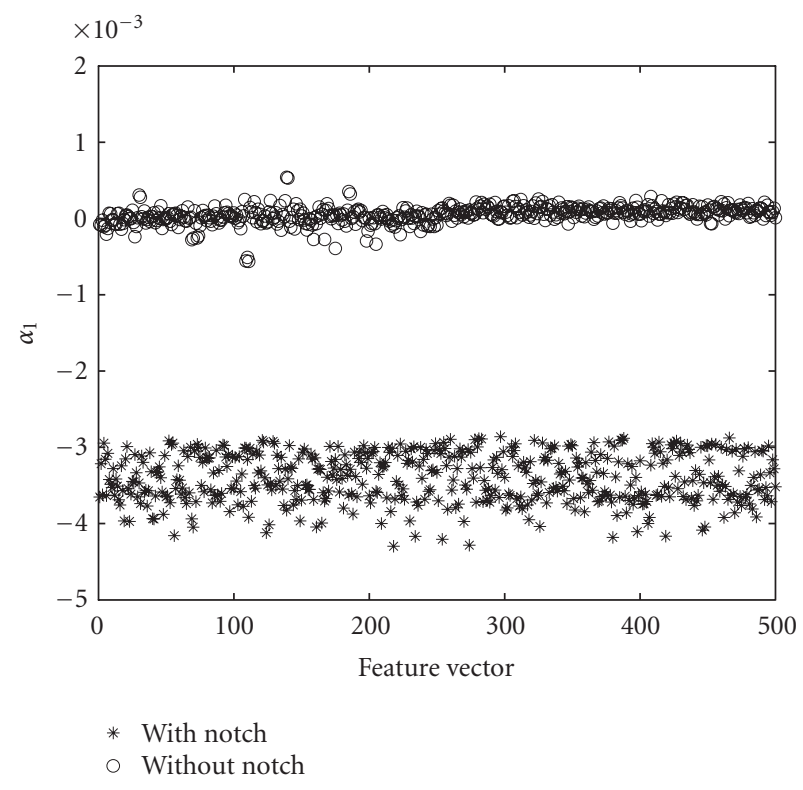

FIgURE 24: HOS-based features extracted from $\mathbf{u}$ to classify the notch disturbance, $\alpha=\widetilde{c}_{2, u}(97)$.

Table 3: Performance of the proposed technique for disturbance classification in the vector $\mathbf{u}$ when $N=1024$ is considered.

\begin{tabular}{l|c}
\hline Disturbance & CR in \% \\
\hline notch & 100 \\
Impulsive transient & 100 \\
Oscillatory transient (damped oscillation) & 100 \\
\hline
\end{tabular}

the approximations to estimate the HOS-based features with (35)-(37) provide better results than those obtained with approximations provided by (31)-(34); (iii) the combination of features obtained with different approximations provides better separability; (iv) the use of cumulants extracted from the ordered vectors usually does not offer any advantage over the usual cumulants; (v) the cumulants associated with 3rd-order are useless for disturbance classification; (vi) in the majority of the cases, the Baysian classifiers reduce to linear classifiers because there is a linear separability between the classes; (vii) to classify seven disturbances, we have to evaluate only eight HOS-based features.

Table 4 presents the performance of the proposed technique applied to classify multiple primitive patterns. Here, it is considered $N=1024$. In Table 4 , the term Transient refers to notch, spike, or damped oscillation, the term Fund denotes disturbances in the fundamental component, the term Harm refers to the harmonic disturbance, and the term Two transients and Three Transients mean that two or three distinct transients occur at the same frame, respectively. From Table 4, one verifies that the proposed technique is capable of
TABle 4: Performance of the proposed technique for the classification of multiple disturbances in vector $\mathbf{x}$.

\begin{tabular}{l|c}
\hline Disturbances & CR in \% \\
\hline Fund + Harm & 100 \\
Fund + Harm + One Transient & 99.98 \\
Fund + Harm + Two Transients & 98.35 \\
Fund + Harm + Three Transients & 96.89 \\
\hline
\end{tabular}

classifying several sets of multiple primitive patterns in voltage signals.

\subsection{Performance comparison}

Finally, the proposed technique is compared against a previous technique recently introduced in [2]. A set of disturbances, defined in accordance with [3] and reproduced in Table 5, was generated by following the same procedure adopted in $[2,3]$. By using this set of disturbances, a performance comparison between the technique discussed in [2] and the one introduced in this contribution was carried out and the results are comparatively reported in Table 6 . Based on the reported results, one can verify that the proposed technique not only surpasses the performance of the previous one, but it also shows a considerable improvement.

One has to note that the results reported in both Sections 4.1 and 4.2 were obtained with disturbances synthetically generated. As a result, the proposed technique will show a degradation in its performance if it is applied to real voltage signals, but it is worth stating that the classification technique proposed in $[2,3]$ will present similar behavior. At this point, we are not able to say, under this situation, what technique will present the lowest performance degradation.

\section{CONCLUSIONS}

In this contribution, a paradigm and a technique to classify single and multiple disturbances in electric signals are introduced. The main advantage offered by the paradigm is the use of the principle of divide to conquer to decompose the powerline signals into a set of primitive components in which simple and powerful feature extraction, feature selection, and classification techniques can be applied to recognize primitive patterns (single and multiple disturbances).

Based on the proposed paradigm, a disturbance classification technique is presented to classify single and the most probable sets of multiple disturbances in voltage signals. The numerical results obtained with computational simulations indicate that the proposed technique shows considerable improvement in terms of classification ratio.

At the moment, some research is being carried out to include flicker, interharmonic, unbalances, and exponential decaying as primitive patterns. 
TABLE 5: Power quality disturbance models following [3].

\begin{tabular}{|c|c|c|c|}
\hline PQ disturbance & Class & Model & Parameters \\
\hline Normal & $\mathrm{C} 1$ & $x(t)=A_{0} \sin \left(\omega_{0} t\right)$ & $A_{0}=1$ \\
\hline Swell & $\mathrm{C} 2$ & $\begin{array}{l}x(t)=A_{0}\left\{1+\alpha\left[u\left(t-t_{1}\right)-u\left(t-t_{2}\right)\right]\right\} \sin \left(\omega_{0} t\right) \\
t_{1}<t_{2}, u(t)=\left\{\begin{array}{l}1, t \geq 0 \\
0, t<0\end{array}\right.\end{array}$ & $\begin{array}{l}0.1 \leq \alpha \leq 0.8 \\
T \leq t_{2}-t_{1} \leq 9 T\end{array}$ \\
\hline Sag & $\mathrm{C} 3$ & $x(t)=A_{0}\left\{1-\alpha\left[u\left(t-t_{1}\right)-u\left(t-t_{2}\right)\right]\right\} \sin \left(\omega_{0} t\right)$ & $0.1 \leq \alpha \leq 0.8, T \leq t_{2}-t_{1} \leq 9 T$ \\
\hline Harmonic & $\mathrm{C} 4$ & $\begin{aligned} x(t)=A_{0}[ & \alpha_{1} \sin \left(\omega_{0} t\right)+\alpha_{3} \sin \left(3 \omega_{0} t\right) \\
& \left.+\alpha_{5} \sin \left(5 \omega_{0} t\right)+\alpha_{7} \sin \left(7 \omega_{0} t\right)\right]\end{aligned}$ & $\begin{array}{l}0.05 \leq \alpha_{3} \leq 0.15,0.05 \leq \alpha_{5} \leq 0.15 \\
0.05 \leq \alpha_{7} \leq 0.15, \sum \alpha_{i}^{2}=1\end{array}$ \\
\hline Outage & C5 & $x(t)=A_{0}\left\{1-\alpha\left[u\left(t-t_{1}\right)-u\left(t-t_{2}\right)\right]\right\} \sin \left(\omega_{0} t\right)$ & $0.9 \leq \alpha \leq 0.1, T \leq t_{2}-t_{1} \leq 9 T$ \\
\hline Sag + Harmonic & C6 & $\begin{aligned} x(t)=A_{0}\{1-\alpha[ & u\left(t-t_{1}\right)- \\
& \\
\left.\left.u\left(t-t_{2}\right)\right]\right\} & {\left[\alpha_{0} \sin \left(\omega_{0} t\right)\right.} \\
& \left.+\alpha_{3} \sin \left(3 \omega_{0} t\right)+\alpha_{5} \sin \left(5 \omega_{0} t\right)\right]\end{aligned}$ & $\begin{array}{l}0.1 \leq \alpha \leq 0.9, T \leq t_{2}-t_{1} \leq 9 T \\
0.05 \leq \alpha_{3} \leq 0.15,0.05 \leq \alpha_{5} \leq 0.15, \sum \alpha_{i}^{2}=1\end{array}$ \\
\hline Swell + Harmonic & $\mathrm{C} 7$ & $\begin{aligned} x(t)=A_{0}\{1+\alpha[ & u\left(t-t_{1}\right) \\
\left.\left.-u\left(t-t_{2}\right)\right]\right\}[ & \alpha_{0} \sin \left(\omega_{0} t\right) \\
& \left.+\alpha_{3} \sin \left(3 \omega_{0} t\right)+\alpha_{5} \sin \left(5 \omega_{0} t\right)\right]\end{aligned}$ & $\begin{array}{l}0.1 \leq \alpha \leq 0.9, T \leq t_{2}-t_{1} \leq 9 T \\
0.05 \leq \alpha_{3} \leq 0.15,0.05 \leq \alpha_{5} \leq 0.15, \sum \alpha_{i}^{2}=1\end{array}$ \\
\hline
\end{tabular}

TABle 6: Performance comparison in terms of classification ratio (\%) achieved by proposed technique and the technique proposed in [2].

\begin{tabular}{c|cc}
\hline Class & Proposed technique & Technique proposed in [2] \\
\hline C1 & 100 & 100 \\
C2 & 100 & 100 \\
C3 & 100 & 85.55 \\
C4 & 100 & 100 \\
C5 & 100 & 82 \\
C6 & 100 & 96.50 \\
C7 & 100 & 100 \\
\hline
\end{tabular}

\section{ACKNOWLEDGMENTS}

This work was supported in part by CNPq under Grants 150064/2005-5 and 550178/2005-8 and FAPEMIG under Grant TEC 00181/06, all from Brazil.

\section{REFERENCES}

[1] M. V. Ribeiro, Signal processing techniques for power line communication and power quality applications, Ph.D. thesis, Department of Communications, University of Campinas, Sao Paulo, Brazil, April 2005.

[2] H. He and J. A. Starzyk, "A self-organizing learning array system for power quality classification based on wavelet transform," IEEE Transactions on Power Delivery, vol. 21, no. 1, pp. 286-295, 2006.

[3] T. K. Abdel-Galil, M. Kamel, A. M. Youssef, E. F. El-Saadany, and M. M. A. Salama, "Power quality disturbance classification using the inductive inference approach," IEEE Transactions on Power Delivery, vol. 19, no. 4, pp. 1812-1818, 2004.

[4] T. X. Zhu, S. K. Tso, and K. L. Lo, "Wavelet-based fuzzy reasoning approach to power-quality disturbance recognition," IEEE
Transactions on Power Delivery, vol. 19, no. 4, pp. 1928-1935, 2004.

[5] M. Wang and A. V. Mamishev, "Classification of power quality events using optimal time-frequency representations-part 1: theory," IEEE Transactions on Power Delivery, vol. 19, no. 3, pp. 1488-1495, 2004.

[6] M. S. Azam, F. Tu, K. R. Pattipati, and R. Karanam, "A dependency model-based approach for identifying and evaluating power quality problems," IEEE Transactions on Power Delivery, vol. 19, no. 3, pp. 1154-1166, 2004.

[7] M. Wang, G. I. Rowe, and A. V. Mamishev, "Classification of power quality events using optimal time-frequency representations-part 2: application," IEEE Transactions on Power Delivery, vol. 19, no. 3, pp. 1496-1503, 2004.

[8] Z.-L. Gaing, "Wavelet-based neural network for power disturbance recognition and classification," IEEE Transactions on Power Delivery, vol. 19, no. 4, pp. 1560-1568, 2004.

[9] J. Chung, E. J. Powers, W. M. Grady, and S. C. Bhatt, "Power disturbance classifier using a rule-based method and wavelet packet-based hidden Markov model," IEEE Transactions on Power Delivery, vol. 17, no. 1, pp. 233-241, 2002.

[10] W. R. A. Ibrahim and M. M. Morcos, "A power quality perspective to system operational diagnosis using fuzzy logic and adaptive techniques," IEEE Transactions on Power Delivery, vol. 18, no. 3, pp. 903-909, 2003.

[11] I. W. C. Lee and P. K. Dash, "S-transform-based intelligent system for classification of power quality disturbance signals," IEEE Transactions on Industrial Electronics, vol. 50, no. 4, pp. 800-805, 2003.

[12] P. K. Dash, B. K. Panigrahi, D. K. Sahoo, and G. Panda, "Power quality disturbance data compression, detection, and classification using integrated spline wavelet and S-transform," IEEE Transactions on Power Delivery, vol. 18, no. 2, pp. 595-600, 2003.

[13] J. Huang, M. Negnevitsky, and D. T. Nguyen, "A neural-fuzzy classifier for recognition of power quality disturbances," IEEE Transactions on Power Delivery, vol. 17, no. 2, pp. 609-616, 2002. 
[14] A. M. Gaouda, S. H. Kanoun, M. M. A. Salama, and A. Y. Chikhani, "Wavelet-based signal processing for disturbance classification and measurement," IEE Proceedings: Generation, Transmission and Distribution, vol. 149, no. 3, pp. 310-318, 2002.

[15] M. Kezunovic and Y. Liao, "A novel software implementation concept for power quality study," IEEE Transactions on Power Delivery, vol. 17, no. 2, pp. 544-549, 2002.

[16] J. Y. Lee, Y. J. Won, J.-M. Jeong, and S. W. Nam, “Classification of power disturbance using feature extraction in timefrequency plane," Electronics Letters, vol. 38, no. 15, pp. 833835, 2002.

[17] T. A. Hoang and D. T. Nguyen, "Improving training of radial basis function network for classification of power quality disturbances," Electronics Letters, vol. 38, no. 17, pp. 976-977, 2002.

[18] J. V. Wijayakulasooriya, G. A. Putrus, and P. D. Minns, "Electric power quality disturbance classification using selfadapting artificial neural networks," IEE Proceedings: Generation, Transmission and Distribution, vol. 149, no. 1, pp. 98-101, 2002.

[19] E. Styvaktakis, M. H. J. Bollen, and I. Y. H. Gu, "Expert system for classification and analysis of power system events," IEEE Transactions on Power Delivery, vol. 17, no. 2, pp. 423-428, 2002.

[20] S. Santoso, W. M. Grady, E. J. Powers, J. Lamoree, and S. C. Bhatt, "Characterization of distribution power quality events with Fourier and wavelet transforms," IEEE Transactions on Power Delivery, vol. 15, no. 1, pp. 247-254, 2000.

[21] M. Kezunovic and Y. Liao, "A new method for classification and characterization of voltage sags," Electric Power Systems Research, vol. 58, no. 1, pp. 27-35, 2001.

[22] P. K. Dash, R. K. Jena, and M. M. A. Salama, "Power quality monitoring using an integrated Fourier linear combiner and fuzzy expert system," International Journal of Electrical Power \& Energy System, vol. 21, no. 7, pp. 497-506, 1999.

[23] A. M. Gaouda, M. M. A. Salama, M. R. Sultan, and A. Y. Chikhani, "Power quality detection and classification using wavelet-multiresolution signal decomposition," IEEE Transactions on Power Delivery, vol. 14, no. 4, pp. 1469-1476, 1999.

[24] S.-J. Huang, C.-T. Hsieh, and C.-L. Huang, "Application of wavelets to classify power system disturbances," Electric Power Systems Research, vol. 47, no. 2, pp. 87-93, 1998.

[25] C. H. Lee and S. W. Nam, "Efficient feature vector extraction for automatic classification of power quality disturbances," Electronics Letters, vol. 34, no. 11, pp. 1059-1061, 1998.

[26] J. S. Lee, C. H. Lee, J. O. Kim, and S. W. Nam, "Classification of power quality disturbances using orthogonal polynomial approximation and bispectra," Electronics Letters, vol. 33, no. 18, pp. 1522-1524, 1997.

[27] A. K. Ghosh and D. L. Lubkeman, "The classification of power system disturbance waveforms using a neural network approach," IEEE Transactions on Power Delivery, vol. 10, no. 1, pp. 109-115, 1995.

[28] A. M. Youssef, T. K. Abdel-Galil, E. F. El-Saadany, and M. M. A. Salama, "Disturbance classification utilizing dynamic time warping classifier," IEEE Transactions on Power Delivery, vol. 19, no. 1, pp. 272-278, 2004.

[29] C. A. Duque, M. V. Ribeiro, F. R. Ramos, and J. Szczupak, "Power quality event detection based on the divide and conquer principle and innovation concept," IEEE Transactions on Power Delivery, vol. 20, no. 4, pp. 2361-2369, 2005.
[30] M. V. Ribeiro and J. L. R. Pereira, "An efficient method for the classification of isolated and multiple disturbances in power line signals," in Proceedings of the 12th International Conference on Harmonics and Quality of Power (ICHQP '06), Cascais, Portugal, October 2006.

[31] D. D. Ferreira, A. S. Cerqueira, M. V. Ribeiro, and C. A. Duque, "HOS-based method for power quality event classification," in Proceedings of the 14th European Signal Processing Conference (EUSIPCO '06), vol. 1, pp. 200-206, Florence, Italy, September 2006.

[32] A. S. Cerqueira, M. V. Ribeiro, C. A. Duque, and D. D. Ferreira, "Power quality events recognition using a SVM-based method," to appear in Electric Power System Research.

[33] H. L. van Trees, Detection, Estimation and Modulation Theory, Part I, Springer, New York, NY, USA, 1968.

[34] H. L. van Trees, Detection, Estimation and Modulation Theory, Part III, Springer, New York, NY, USA, 1971.

[35] R. N. McDonough and A. D. Whalen, Detection of Signals in Noise, Academic Press, London, UK, 1995.

[36] IEEE 519 Working Group, “IEEE 519-1995 recommended practices and requirements for harmonic control in electrical power systems institute of electrical and electronics engineers," May 1992.

[37] J. R. Carvalho, P. H. Gomes, A. S. Cerqueira, M. V. Ribeiro, C. A. Duque, and J. Szczupak, "PLL based multirate harmonic estimation," in IEEE PES General Meeting, Tampa, Fla, USA, 2007.

[38] M. V. Ribeiro, C. A. G. Marques, C. A. Duque, A. S. Cerqueira, and J. L. R. Pereira, "Detection of disturbances in voltage signals for power quality analysis using HOS," EURASIP Journal on Advances in Signal Processing, vol. 2007, Article ID 59786, 13 pages, 2007.

[39] J. Kauraniemi, T. I. Laakso, I. Hartimo, and S. J. Ovaska, “Delta operator realizations of direct-form IIR filters," IEEE Transactions on Circuits and Systems II, vol. 45, no. 1, pp. 41-52, 1998.

[40] R. H. Middleton and G. C. Goodwin, "Improved finite word length characteristics in digital control using delta operators," IEEE Transactions on Automatic Control, vol. 31, no. 11, pp. 1015-1021, 1986.

[41] A. K. Jain, R. P. W. Duin, and J. Mao, "Statistical pattern recognition: a review," IEEE Transactions on Pattern Analysis and Machine Intelligence, vol. 22, no. 1, pp. 4-37, 2000.

[42] J. M. Mendel, “Tutorial on higher-order statistics (spectra) in signal processing and system theory: theoretical results and some applications," Proceedings of the IEEE, vol. 79, no. 3, pp. 278-305, 1991.

[43] C. Nikias and J. Mendel, "Signal processing with higher-order statistics," IEEE Transactions on Signal Processing, vol. 41, no. 1, pp. 10-38, 1999.

[44] C. L. Nikias and A. P. Petropulu, Hiher-Order Spectra Analysis-A Nonlinear Signal Processing Framework, PrenticeHall, Englewood Cliffs, NJ, USA, 1993.

[45] M. Frisch and H. Messer, "Transient signal detection using prior information in the likelihood-ratio-test," IEEE Transactions on Signal Processing, vol. 41, no. 6, pp. 2177-2192, 1993.

[46] Ö. N. Gerek and D. G. Ece, "Power-quality event analysis using higher order cumulants and quadratic classifiers," IEEE Transactions on Power Delivery, vol. 21, no. 2, pp. 883-889, 2006.

[47] B. Picinbono, "Polyspectra of ordered signals," IEEE Transactions on Information Theory, vol. 45, no. 7, pp. 2239-2252, 1999. 
[48] S. Theodoridis and K. Koutroumbas, Pattern Recognition, Academic Press, San Diego, Calif, USA, 1999.

[49] IEEE 1159 Working Group, "IEEE 1159-1995 recommended practice on monitoring electrical power quality," November 1995.

Moisés Vidal Ribeiro was born in Três Rios, Brazil, in 1974. He received the B.S. degree in electrical engineering from the Federal University of Juiz de Fora (UFJF), Juiz de Fora, Brazil, in 1999, and the M.S. and Ph.D. degrees in electrical engineering from the University of Campinas (UNICAMP), Campinas, Brazil, in 2001 and 2005, respectively. Currently, he is an Assistant Professor at UFJF. Dr. Ribeiro was a Visiting Re-

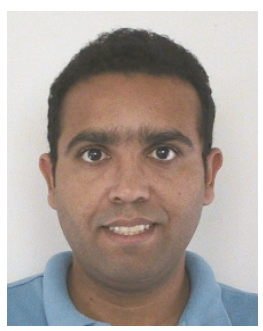
searcher in the Image and Signal Processing Laboratory of the University of California, Santa Barbara, in 2004, a Postdoctoral Researcher at UNICAMP, in 2005, and at UFJF from 2005 to 2006 . He is guest editor for the Special Issues on Emerging Signal Processing Techniques for Power Quality Applications and on Advanced Signal Processing and Computational Intelligence Techniques for Power Line Communications for the EURASIP Journal on Applied Signal Processing and reviewer of international journals. He has been author of 15 journals and 41 conference papers, and holds six patents. His research interests include computational intelligence, digital and adaptive signal processing, power quality, powerline communication, and digital communications. Dr. Ribeiro received student awards from IECON'01 and ISIE'03. He is a Member of the technical program committee of the ISPLC'06, ISPLC'07, CERMA'06, and ANDESCOM'06, and a Member of the IEEE ComSoc Technical Committee on Power Line Communications.

José Luiz Rezende Pereira received his B.S. in 1975 from Federal University of Juiz de Fora, Brazil, the M.S. in 1978 from COPPEFederal University of Rio de Janeiro, and the Ph.D. degree in 1988 from UMIST, UK. From 1977 to 1992, he worked at Federal University of Rio de Janeiro. Since 1993 he has been working at Electrical Engineering Department of Federal University of Juiz de Fora. Dr. Pereira's research interests include

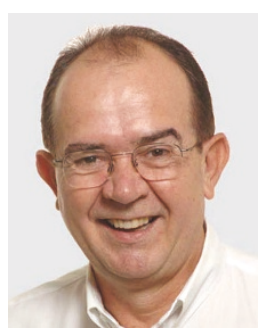
planning and operation modeling for transmission and distribution systems. 